Revue internationale P.M.E.

Économie et gestion de la petite et moyenne entreprise

\title{
La carte cognitive peut-elle constituer un outil fiable pour l'identification des logiques d'action de dirigeants des entreprises familiales tunisiennes?
}

\section{Henda Ellouze Karray}

Volume 19, numéro 2, 2006

URI : https://id.erudit.org/iderudit/1008494ar

DOI : https://doi.org/10.7202/1008494ar

Aller au sommaire du numéro

Éditeur(s)

Presses de l’Université du Québec

ISSN

0776-5436 (imprimé)

1918-9699 (numérique)

Découvrir la revue

Citer cet article

Ellouze Karray, H. (2006). La carte cognitive peut-elle constituer un outil fiable pour l'identification des logiques d'action de dirigeants des entreprises familiales tunisiennes? Revue internationale P.M.E., 19(2), 9-47. https://doi.org/10.7202/1008494ar
Résumé de l'article

Dans cet article, l'auteure se propose de vérifier si la carte cognitive peut constituer un outil fiable pour identifier les logiques d'action de dirigeants d'entreprises familiales. À cet égard, elle examinera d'abord les cartes cognitives et leurs utilisations dans le champ du management pour ensuite présenter les différentes logiques d'action.

Pour vérifier cette hypothèse, elle se penchera sur sept cas de dirigeants d'entreprises familiales tunisiennes appartenant au secteur de l'industrie chimique. La spécificité de la nature de l'entreprise familiale pousse l'auteure à s'y intéresser et à connaître les choix stratégiques de ces dirigeants. 


\title{
La carte cognitive peut-elle constituer un outil fiable pour l'identification des logiques d'action de dirigeants des entreprises familiales tunisiennes?
}

Henda ELLOUZE KARRAY

Université de Sfax

MOTS CLÉS

Entreprise familiale - Carte cognitive - Logiques d'action

\section{RÉSUMÉ}

Dans cet article, l'auteure se propose de vérifier si la carte cognitive peut constituer un outil fiable pour identifier les logiques d'action de dirigeants d'entreprises familiales. À cet égard, elle examinera d'abord les cartes cognitives et leurs utilisations dans le champ du management pour ensuite présenter les différentes logiques d'action.

Pour vérifier cette hypothèse, elle se penchera sur sept cas de dirigeants d'entreprises familiales tunisiennes appartenant au secteur de l'industrie chimique. La spécificité de la nature de l'entreprise familiale pousse l'auteure à s'y intéresser et à connaître les choix stratégiques de ces dirigeants.

\begin{abstract}
The purpose of this article is to verify whether the cognitive map can be considered as reliable to study the action logics of family-business managers. First, it will examine cognitive maps and their use in the management field. Then, it will present different action logics.
\end{abstract}

\section{L'AUTEURE}

Henda Ellouze KarRay est titulaire d'un doctorat en sciences de gestion de l'Université de Montpellier I, Équipe de recherche sur la firme et l'industrie. Elle est assistante titulaire à l'Institut des hautes études commerciales de l'Université de Sfax, en Tunisie. Ses recherches portent sur le thème des entreprises familiales et les logiques d'action des dirigeants. Adresse: Henkel Extra Colle, route de Gabès Km 3, B.P. 144, 30 28, Sfax, Tunisie. Téléphone: 00216983165 38, télécopieur: 00216744530 86. Courriel: <ellouzehenda@yahoo.fr>. 
This research will try to verify this hypothesis by studying the behaviour of seven different family business managers working in the Tunisian chemistry sector. The specific nature of family enterprise drives the author to give a special attention and interest to the understanding of the strategic choices of these managers.

\section{RESUMEN}

Este articulo nos propone verifier si la carta cognitiva prede constituir un instrumento fiable para identificar los logicos de acciones. Respecto a eso, los dirigentes de empresas familiales nos examinaran, de una parte las centras cognitivas y sus utilizaciones en el campo de manajamiento y nos presentaren, de oltra parte los diferentes logicos de acciones.

Esta investigación tentara verifiar nuestro problemàtica a partir de siete casos de dirigentes de impresas familiales tunecinos que forman parte de los sectores de la industria quimica. La especificidad de la naturaleza de la empresa familial nos empuja a interesarse y conocer las elecciones estratégicos de sus dirigentes.

\section{ZUSAMMENFASSUNG}

Der vorliegende Artikel versucht nachzuprüfen, ob die mentale Repräsentation ein brauchbares Instrument zur Identifikation von logischen Aktionen von Führungskräften in Familienunternehmen darstellt. Zu diesem Zweck werden erstens die Formen von mentalen Repräsentationen in der Managementumgebung und zweitens die verschiedenen logischen Aktionen präsentiert.

Anhand von sieben Fallstudien tunesischer Familienunternehmen aus der chemischen Industrie versucht die Studie die oben genannte Problematik zu untersuchen. Das spezifische Wesen der Familienunternehmung ruft ein besonderes Interesse hervor, sich für die strategischen Entscheide der Führungskräfte zu interessieren und sie auch zu verstehen.

\section{Introduction}

Depuis les travaux d'Axelrod (1976) et son équipe dans le domaine de la politique internationale, l'intérêt suscité par le concept de carte cognitive est croissant, notamment en sciences de l'administration, en management stratégique (Cossette et Audet, 1994) et dans plusieurs autres domaines, tels que les systèmes d'informations organisationnels et encore en macroéconomie (Cossette et Audet, 1994).

Le concept de carte cognitive a été au cœur de certaines études, ce qui a donné lieu à plusieurs définitions à ce concept et à maintes utilisations. Ces études, qui ont porté essentiellement sur les divers usages de la carte cognitive, n'ont pas été menées dans des contextes d'entreprises familiales. 
Dans cette recherche, nous tenterons de vérifier une nouvelle utilisation de la carte cognitive par l'étude de dirigeants tunisiens propriétaires d'entreprises familiales. Marchesnay (1997, p. 93) précise que «les études sur l'entrepreneur (lato sensu) font apparaître une extrême diversité d'aspirations, de représentations et de comportements». Il affirme que «sous l'effet des multiples mutations qui ont affecté le monde des entreprises, un mode unique de dirigeant n'est plus possible comme autrefois». Nous supposons que ces dirigeants adoptent des logiques d'action différentes, c'est ce que nous allons vérifier à travers les cartes cognitives de ces derniers. Dans la littérature, la question des logiques d'action a été abordée directement ou indirectement chaque fois que les chercheurs proposent et/ou analysent les typologies d'entrepreneurs existantes ou des typologies nouvelles telles qu'elles sont proposées par Laufer (1975), Gresle (1978), Miles et Snow (1978), Dunkelberg et Cooper (1982), Schein (1983), Bauer (1993), Julien et Marchesnay (1996) et Marchesnay (2003a).

Il faut par ailleurs souligner que la plupart des travaux qui ont étudié les dirigeants, les dirigeants-propriétaires, leurs comportements stratégiques et leurs logiques d'action se sont réalisés dans le cadre de PME et non pas dans le cadre d'entreprises familiales. Une brève distinction entre ces deux concepts peut être utile. Dans une étude portant sur l'entreprise familiale en Europe, Couret (1993) illustre que «la toute petite entreprise ou TPE en langage managérial est par nature une entreprise familiale. Les petites et moyennes entreprises sont des entreprises familiales dans des proportions considérables». À ce propos, Catry et Buff (1996, p. 19) précisent «qu'un grand nombre de sociétés familiales sont des PME, mais les PME ne sont pas toutes des sociétés familiales». Ces auteurs remarquent que l'éventail des entreprises familiales est large puisqu'il englobe des petites entreprises familiales artisanales, des PME régionales, des entreprises d'envergure nationale et même des groupes internationaux, sans oublier certains grands groupes multinationaux d'aujourd'hui qui sont issus d'entreprises familiales et qui continuent à être influencés par les descendants directs des fondateurs.

À travers cette distinction entre la PME et l'entreprise familiale, nous précisons que dans cette recherche nous allons nous intéresser aux PME tunisiennes de nature familiale puisqu'en Tunisie aucune loi ne différencie une entreprise familiale d'une entreprise non familiale. Cela veut dire que l'entreprise sous contrôle familial peut s'étendre de l'entreprise individuelle à la multinationale et peut recouvrir toutes les formes de sociétés: SARL, SA, société en commandite par actions, etc.

Depuis 1953, divers travaux ont abordé le thème de l'entreprise familiale dans des domaines différents, ce qui a donné lieu à plusieurs définitions de l'entreprise familiale, selon la propriété du capital, le type de dirigeant, le contrôle, la possession du capital, etc. Ainsi, devant l'absence de consensus sur une définition de l'entreprise familiale, nous avons retenu celle que nous considérons comme 
la plus globalisante sur le plan des critères, qui nous servira pour l'identification des entreprises familiales tunisiennes. Il s'agit de la définition de Astrachan et Kolenko (1994, p. 251-262): «la famille doit détenir au moins 50\% du capital pour les entreprises ne faisant pas appel à l'épargne publique et $10 \%$ dans le cas inverse - l'entreprise doit, en outre, avoir 10 années d'activité continue - plus d'un membre de la famille doit être impliqué dans ces activités». Enfin, ces auteurs ajoutent un critère, celui de la volonté du propriétaire de transmettre l'entreprise à la prochaine génération.

Compte tenu de la nature spécifique de l'entreprise familiale, connue pour son comportement stratégique conservateur en raison de la structure de la propriété du capital légué par les fondateurs, nous avons jugé intéressant de nous focaliser sur ce type d'entreprise sur le marché tunisien réputé comprendre un grand nombre d'entreprises familiales pour atteindre un double objectif. Nous allons vérifier, d'une part, l'existence de différentes logiques d'action chez les dirigeants étudiés et, d'autre part, l'usage de la carte cognitive en tant qu'outil pertinent pour identifier ces logiques d'action.

\section{Un aperçu théorique sur les concepts clés}

Nous présenterons d'abord l'outil cartographique à travers un bref aperçu sur la littérature et, ensuite, nous établirons les différentes typologies des dirigeants en fonction de leurs choix stratégiques et de leurs logiques d'action.

\subsection{La carte cognitive}

Nous trouvons pour la notion de «carte cognitive» plusieurs définitions en parcourant les différentes recherches. Ainsi, O'Keefe et Nadel (1978) définissent la carte cognitive comme étant «un ensemble d'informations inter-reliées. Elle aide à percevoir des environnements à grande échelle au-delà du rang de la perception immédiate»; ils précisent «qu'il existe une relation entre action et carte. Quand un individu construit des cartes cognitives, il part de ses résultats, de ses petites expériences et tire des conséquences résultant de leurs propres actions ou expériences » (Rakotoarivelo, 1995, p. 6). D'autres chercheurs élaborent une carte cognitive en tant qu'un modèle général de prise de connaissance. Les travaux de Weick et Bougon (1986, p. 106) illustrent cette tendance exprimée dans la citation suivante «elle (la carte cognitive) est une représentation de cette connaissance. Elle se compose des concepts et des relations qu'un participant utilise pour comprendre des situations organisationnelles».

Eden (1988, p. 8 et 11) précise qu' «une carte cognitive est un modèle conçu pour représenter la façon dont une personne définit un problème particulier. Celle-ci n'est pas un modèle général de la façon de penser de quelqu'un [...] La cartographie 
cognitive est un "outil de négociation" qui facilite la réflexion et la prise de décision». Eden, Ackermann et Cropper (1992) se servent de la représentation graphique comme une technique autant pour favoriser l'expression et la négociation d'idées entre individus que pour en permettre une analyse qu'il juge difficilement réalisable autrement. Une carte cognitive constitue alors «une figure dont la visualisation est susceptible d'aider à réfléchir, c'est-à-dire à explorer, à prendre conscience et à transformer ou confirmer des idées plus ou moins partagées» (Cossette, 1994a, p. 7). Cet auteur propose une définition d'ordre général selon laquelle la carte cognitive est «une représentation graphique de la représentation mentale que le chercheur se fait d'un ensemble de représentations discursives énoncées par un sujet à partir de ses propres représentations cognitives, à propos d'un objet particulier» (Cossette, 1994a, p. 9). Elle est ainsi considérée comme étant «une figure tracée par un chercheur pour représenter les affirmations d'un sujet. Elle se compose de deux éléments: des concepts, le plus souvent des variables et des liens, généralement dits de causalité, unissant certains d'entre eux» (Cossette, 1994a, p. 6). Nous adoptons la définition de Cossette (1994a) de la carte cognitive que nous considérons la plus descriptive de ce moyen. Cette définition renseigne sur le contenu des cartes du point de vue concepts, liens et idées exprimées.

Rakotoarivelo (1995, p. 8) précise qu' «une carte peut être distinguée selon son rôle, sa nature et son utilisation. Si l'on s'intéresse tout particulièrement à son utilisation, ceci conduit à distinguer deux façons majeures d'appréhender la carte; soit comme un produit, soit comme un outil». Dans cette recherche, nous considérons la carte comme un outil dont nous cherchons à vérifier l'utilisation.

Par ailleurs, si nous cherchons à connaître les divers niveaux d'analyse, nous pouvons citer les quatre distingués par Jenkins (1994). Le premier porte sur la dynamique interne de la carte. Le deuxième niveau d'analyse concerne le changement de cartes d'un individu, d'un groupe ou d'une organisation dans le temps et dans différents contextes, dans divers secteurs ou pays; le but est d'établir des qualités prédites des cartes. Le troisième niveau s'intéresse au rôle orienté action des cartes cognitives et, enfin, le quatrième vise la comparaison directe des cartes de différents individus et de différents groupes. De ces quatre niveaux d'analyse, nous avons choisi le premier niveau proposé par Jenkins (1994) et qui a été notamment suivi par Bougon (1987) et Cossette et Audet (1994).

La carte cognitive peut être élaborée soit pour un individu, soit pour un groupe, soit pour une organisation. Dans cette recherche, étant donné les deux objectifs perçus, nous allons réaliser l'entretien avec les dirigeants des entreprises familiales repérées. Pendant l'entretien et au fur et à mesure que les dirigeants tiennent leurs discours, nous allons commencer à tracer les premières versions des cartes cognitives individuelles des dirigeants. Il est à noter qu'il existe différentes formes de présentation des cartes cognitives. Rakotoarivelo (1995, p. 12) précise 
que «la carte cognitive peut être, premièrement sous forme de représentation graphique: circulaire (Bougon, Weick et Binkhorst, 1977), horizontale (Axelrod, 1976), verticale (Eden, Ackermann et Cropper, 1992) et représentation de deux cartes de deux individus sur des axes (Bowman et Johnson, 1991). Deuxièmement, sous forme matricielle et, troisièmement, sous forme de sous-ensembles mathématiques». Cet auteur note aussi que «le choix du formalisme dépend de l'utilisation de la carte: il est plus facile de visualiser une carte sous forme graphique que sous forme matricielle et/ou sous forme de sous-ensembles mathématiques et qu'il est plus facile d'effectuer les tests statistiques et les calculs avec la forme matricielle et les sous-ensembles mathématiques» (Rakotoarivelo, 1995, p. 15).

Nous avons choisi de représenter graphiquement les cartes cognitives des dirigeants. Nous nous sommes inspirée de la carte de type causal de Eden, Ackermann et Cropper (1992) où les concepts sont classés verticalement en bas pour les causes (ou explications, moyens, détails) et en haut pour les effets (ou conséquences, fins). Rakotoarivelo (1995, p. 13) signale, en ce qui concerne ce type de représentation, que «c'est la forme la plus facile pour la manipulation et la comparaison des cartes». De plus, Eden, Ackermann et Cropper (1992) ont utilisé ce type de représentation pour comprendre de quelle manière les managers interagissent avec leur environnement. En nous fondant sur ces deux argumentations, nous avons choisi de nous référer à ce type de carte en convergeant verticalement les concepts utilisés par les dirigeants vers un même concept: «la mondialisation».

Il y a lieu de souligner que «plus le nombre de concepts ou de liens est élevé, plus la carte risque de prendre l'apparence d'un fouillis indescriptible» (Cossette et Audet, 1994, p. 18). De plus, ces auteurs ajoutent que «le discours produit par un sujet dans un contexte d'interlocution particulier à partir de ses propres représentations cognitives guide le chercheur dans la fabrication d'une carte cognitive» (Cossette et Audet, 1994, p. 15). Ils précisent aussi que «le discours du sujet contient des phénomènes de langue comme des présupposés et des pré-construits culturels et s'élabore à l'aide de mécanismes logico-discursifs, parfois difficilement repérables. Pour le chercheur, ces éléments viennent compliquer le décodage du discours et, bien sûr, sa cartographie» (Cossette et Audet, 1994, p. 17). En nous fondant sur ces propos, nous avons choisi, lors de la phase de construction des cartes, de conserver délibérément les idées émises par les dirigeants sous leur forme apparente. Autrement dit, nous avons schématisé dans les cartes le «langage naturel» des dirigeants au sens de Grize (1989). Cette démarche poursuit deux objectifs: d'une part, rester fidèle aux idées émises par les interviewés pour réduire le risque de biais et, d'autre part, permettre une lecture plus rapide et plus claire des relations entre les concepts. Grâce à cette démarche, nous avons obtenu des cartes cognitives lisibles et compréhensibles. 
À travers cette recherche, nous montrerons le rôle que peut jouer la carte cognitive en étant un moyen pertinent pour connaître les logiques d'action des dirigeants en nous référant à leurs choix stratégiques. Bien que l'utilisation de la cartographie cognitive dans le champ de la gestion stratégique soit récente et n'ait fait l'objet que de peu d'études empiriques, nous pouvons citer plusieurs usages attribués à la carte cognitive par différents chercheurs. Cossette (1994a) relève que «l'utilisation des cartes cognitives s'avère très appropriée - tous les "cartographes" de la cognition en conviennent - étant donné qu'elle permet l'analyse d'un réseau de concepts et qu'elle aide à en comprendre la dynamique». Elle peut servir d'outil à la communication, avec soi-même ou avec d'autres. Selon Weick et Bougon (1986), la carte cognitive met en évidence des caractéristiques souvent insoupçonnées du schème représenté, notamment à la suite de l'étude des sentiers et boucles formés par des liens qui relient des concepts. Eden et al. (1981) notent, pour leur part, que la cartographie cognitive est un outil destiné fondamentalement à favoriser le processus de prise de décision stratégique en groupe. Enfin, Cossette (1996) montre que la carte cognitive peut aider le propriétaire-dirigeant de PME à mettre au point sa vision stratégique et à l'analyser.

En nous fondant sur les résultats de ces études, nous devrions être en mesure d'obtenir une vérification de la problématique de cette recherche. Mais auparavant, il convient de rappeler brièvement les méthodes employées par les chercheurs et les usagers de la carte cognitive.

En fait, la majorité des recherches se basent sur l'analyse de données recueillies à partir d'entretiens individuels (Bougon, Weick et Binkhorst, 1977, et Laukkanen, 1994) et/ou de groupe (Langfield-Smith, 1992; Allard-Poési, 1997); d'autres recherches proposent de construire des cartes cognitives sur la base de données issues de documents ${ }^{1}$ internes (journaux d'entreprises ou revues sectorielles), d'archives (rapports annuels d'entreprises), de révélations publiques ou de comptes rendus de discours. Fahey et Narayanan (1989), dans leur étude longitudinale (1960 à 1979) sur l'industrie du récepteur de télévision, construisent des cartes cognitives à partir des rapports annuels adressés aux actionnaires et de déclarations de managers des entreprises Zenith et RCA publiées dans une revue hebdomadaire (Television Digest) et destinées aux professionnels du secteur concerné. De même, Stubbart et Ramaprasad (1988) élaborent les cartes cognitives de deux responsables de l'industrie américaine de l'acier (Roderick, président d'U.S Steel et F.K. Iverson, directeur général de Nucor Steel) à partir de certaines de leurs déclarations publiques, choisies par ce qu'elles contiennent des thèmes stratégiques récurrents, et d'entrevues avec les auteurs. Ils précisent toutefois que

1. La carte cognitive élaborée à partir de documents écrits ou de comptes rendus de réunions publiques a été couramment utilisée dans le domaine des sciences politiques (Axelrod, 1976; Hart, 1976, 1977). 
«ces deux sources d'informations ne génèrent pas le même type de représentations dans la mesure où les déclarations faites à une assemblée ne reflètent pas totalement la pensée des orateurs» (Chabin, 2001, p. 176).

De son côté, Cossette (1994b, 1994c) utilise un questionnaire en profondeur à partir d'une grille d'exploration systématique suivie de la constitution de cartes et de la vérification de leur sens auprès du dirigeant. Cette grille constitue une technique de collecte de matériaux. Cette méthode - qui prend forme, comme nous allons le préciser par la suite - est utilisée aussi par Paradas. Le sujet est invité à indiquer les facteurs importants qui, selon lui, vont avoir un impact sur l'avenir de son entreprise. Chacun de ces facteurs est écrit par le chercheur au centre d'une feuille préparée à cette fin. Ce travail terminé, le chercheur demande au sujet de se focaliser sur le premier facteur stratégique nommé et de déterminer les facteurs qui auront, selon lui, une influence sur ce facteur stratégique ainsi que sur les facteurs qui seront influencés par lui. Toutes ces «explications» et «conséquences» sont consignées aux endroits appropriés. Dans un deuxième temps, le chercheur demande au sujet de déterminer les facteurs ayant un impact sur ceux nouvellement relevés, d'une part, et sur ceux influencés par eux de l'autre. Ce travail d'exploration systématique est répété pour chacun des facteurs stratégiques mentionnés au départ par le dirigeant. À partir des matériaux recueillis dans cette phase, le chercheur peut tracer la carte cognitive de ce dirigeant. Dans une deuxième phase, le chercheur va s'assurer de la crédibilité et de la validité de la carte cognitive tracée. Pour cela, une deuxième rencontre se déroule avec le répondant pour confirmer l'emplacement des concepts et les liens qui relient les différents concepts et, enfin, ajouter et faire des modifications si le chercheur en sent le besoin.

Pour leur part, Nkongolo-Bakenda, d'Amboise et Garnier (1994) recueillent les données en deux phases. La première phase consiste en des entretiens avec les dirigeants enregistrés et retranscrits sur le même type de questionnaire que celui qui a servi de guide à l'entretien. L'élaboration du guide du questionnaire s'est largement inspirée de la littérature relative au concept étudié (d'Amboise et Gasse, 1989). Une fois la phase de l'enquête achevée, une deuxième phase de collecte des données est entamée. Cette phase est réalisée simultanément par questionnaire écrit et entretien dirigé. Enfin, les résultats du dépouillement des données et ceux des analyses subséquentes sont présentés au moyen d'indicateurs de performance. Pour sa part, Allain (1999) se fonde sur une enquête approfondie auprès du répondant à l'aide d'un guide d'entretien permettant de mettre au jour les éléments de la grille d'analyse. Cette grille composée de trois phases pour la résolution d'un problème a été utilisée pour comprendre les décisions de pilotage stratégique de l'entrepreneur agricole (Allain, 1999). D'autres méthodes sont utilisées pour construire les cartes cognitives, que ce soit à partir d'entretiens individuels et/ou de groupe. Bougon (1992) s'appuie sur une approche très structurée (voir la méthode «Self-Q»); 
elle se différencie des recherches basées sur des interviews délibérément ouvertes (voir les travaux de Eden fondés sur la théorie des construits personnels de Kelly²$^{2}, 1955$ et 1963).

La synthèse des diverses recherches qui ont utilisé la carte cognitive montre que la méthode que nous adoptons diffère des autres. En effet, nous avons choisi d'obtenir l'information d'une façon indirecte par le moyen de deux techniques. Nous avons utilisé la technique de l'interview non directive active, fondée sur la technique des commentaires provoqués. La présentation aux dirigeants d'un cas fictif illustrant un problème précis fait que l'interview est ouverte, mais les discours des interviewés sont un peu guidés. Nous reviendrons plus loin sur les détails concernant notre méthodologie.

\subsection{Les choix stratégiques des dirigeants et leurs logiques d'action}

\section{Haddadj et D’Andria (1998, p. 58-59) précisent que}

[...] le dirigeant peut choisir entre quatre stratégies: stratégie orientée vers un nouveau positionnement, en étendant le champ de son marché actuel, en offrant de nouveaux produits et en cherchant de nouveaux clients; stratégie orientée vers un maintien, l'entreprise adoptant cette stratégie est localisée dans un marché où la concurrence est forte et où l'environnement est décrit comme étant instable et intense. Ces auteurs supposent que cette entreprise cherche à réduire les prix des produits en améliorant leur qualité en permanence; stratégie orientée vers un développement externe pour réagir à une compétition intense, et ce, en choisissant de s'ouvrir et de s'internationaliser et, enfin, stratégie orientée vers un développement interne en apportant des changements profonds pour attirer de nouveaux clients, sachant qu'ils considèrent que les besoins des clients actuels sont satisfaits et que le système relationnel établi avec leurs anciens clients est maintenu».

Ces quatre stratégies peuvent être prises par plusieurs types de dirigeants.

Plusieurs typologies ont servi à classer les dirigeants en prenant en considération leurs choix stratégiques. Miles et Snow (1978), en se basant sur l'orientation stratégique adoptée par les dirigeants, ont découvert «le prospecteur, le défenseur, l'analyste et le réactif»:

2. Kelly a publié en 1955 un ouvrage en deux volumes intitulé The Psychology of Personal Constructs. Il invitera le lecteur à porter attention aux fondements philosophiques et théoriques de la théorie des construits personnels en regroupant les premiers chapitres de son ouvrage initial (après avoir effectué certaines modifications pour les rendre plus accessibles dans une publication éditée en 1963 et intitulée: A Theory of Personality. 
- Le prospecteur, par sa prise de risques maximale, serait fortement créateur de valeurs (innovateur systématique); jouit d'une flexibilité et d'une rapidité d'adaptation; scrute son environnement à la recherche d'occasions et poursuit agressivement son expansion sur de nouveaux marchés.

- Le défenseur, par sa prise minimale de risques, serait créateur de valeurs de manière satisfaisante (protection du marché existant); adopte une approche conservatrice; se concentre sur le maintien de sa position compétitive actuelle; n'a pas d'obligation de réponse en ce qui a trait aux changements environnementaux.

- L'analyste, en tant que «maximisateur» ou «opportuniste», récolterait les fruits sans en assumer, de manière relative, l'ensemble des coûts; par la diversification de son domaine d'activité, doit s'engager dans des processus de planification multiples et une évaluation très formalisée de nouvelles opportunités de marché.

- Le réactif, enfin, peut osciller entre création et destruction de valeurs au regard des décalages entre une configuration opportune et la situation réelle de l'entreprise; ne poursuit pas de stratégie spécifique mais réagit à des menaces ou opportunités de compétition d'une manière ad hoc souvent défensive; est passif dans la gestion des problèmes jusqu'à ce qu'il soit contraint par des pressions extérieures à réagir aux situations problématiques.

Aussi, Ivanaj et Géhin (1997) distinguent deux grands comportements types du dirigeant de PME: l'un plutôt managérial (avec une recherche de croissance avec prise de risques) et l'autre plutôt patrimonial (prise de risques très limitée). Ces deux comportements rejoignent la typologie de Julien et de Marchesnay (1988, 1996). Ils distinguent alors le dirigeant de type PIC, Pérennité - Indépendance Croissance, du dirigeant de type CAP, Croissance - Autonomie - Pérennité. Le premier type de dirigeant, PIC, fait référence à un individu dont le principal motif à la création, la reprise ou l'acceptation en héritage d'une entreprise est la volonté d'exercer son métier en dehors du cadre contraignant de l'organisation hiérarchique. Lié à son entreprise par des liens quasi affectifs, son but premier est de faire en sorte que celle-ci se pérennise. En conséquence de sa préférence marquée envers ces deux buts - pérennité et indépendance -, la croissance apparaît reléguée au dernier plan. Ses buts l'incitent en outre à privilégier l'autofinancement ou l'aide familiale. Il obéit ainsi à une logique d'action patrimoniale. Alors que le dirigeant au profil CAP est motivé par la croissance de son entreprise, synonyme pour lui de réussite personnelle et d'accomplissement de soi. Les profits réalisés doivent en partie servir à financer cette croissance, de même qu'à investir dans des occasions d'affaires. Ce dirigeant tient également à rester maître de ses choix dans son entreprise. S'agissant de ses attitudes et comportements, ce dirigeant semble 
trouver de l'attrait aux activités qui se rapportent à la gestion; il trouve important de découvrir de nouveaux marchés. Par ailleurs, pour réaliser ses paris sur l'avenir, le dirigeant CAP serait prêt à aller chercher des capitaux hors de son entourage familial, voire hors endettement en ayant une certaine attirance pour le risque. Il obéit à une logique de valorisation des capitaux engagés.

Pour sa part, Laufer (1975) distingue quatre types d'entrepreneurs: le manager ou l'innovateur, l'entrepreneur-propriétaire orienté vers la croissance et recherchant l'efficacité, et l'entrepreneur artisan. Il précise que «leur motivation diffère sur les plans du désir de réalisation, de croissance, de pouvoir ou d'autonomie et le degré de motivation à créer résulte du degré de contrariété sur l'un ou plusieurs de ces plans» (Verstraete, 1999, p. 78). Au sujet de la cellule familiale, il ajoute qu' «elle joue un rôle important également» (Verstraete, 1999, p. 78). Marchesnay (2003a) propose une autre typologie qui repose sur les logiques d'action et sur les niveaux d'aspirations. Il distingue une logique d'action centrée sur l'accumulation d'actifs (logique de stocks ou patrimoniale) ou sur la recherche de revenus (logique de flux ou de valorisation) et il hiérarchise trois niveaux d'aspiration de la part de l'entrepreneur: l'aspiration à la pérennité $(\mathrm{P})$, à l'indépendance $(\mathrm{I})$ ou l'autonomie $(\mathrm{A})$ et à la croissance $(\mathrm{C})$.

En parlant de logique d'action patrimoniale, Bouhaouala et Chantelat (2002, p. 31) notent que «ce type de dirigeants vise une rentabilité financière qui lui permet de maximiser ses revenus sous la contrainte du maintien de l'entreprise au sein du cercle familial». Ces propos montrent que ce type de dirigeants évite les investissements lourds et incertains pouvant le conduire à une dépendance envers le marché des capitaux. Bouhaouala (1999) note que «la réussite pour les patrimoniaux prend deux dimensions importantes: financière à court terme et moyen terme, "morale" à long terme». Elle est financière à travers l'argent que leur procure leur travail et morale à travers la conservation du patrimoine familial.

Plusieurs auteurs (McClelland, 1965; Laufer, 1975; Carland et al., 1984; Julien et Marchesnay, 1988; Ivanaj et Géhin, 1997; ...) s'accordent sur les spécificités du dirigeant adoptant une logique d'action patrimoniale.

Les dirigeants-managers «qu'ils soient de simples commettants aux ordres de propriétaires, ou eux-mêmes propriétaires-dirigeants, sont animés autant par un besoin de pouvoir que par un désir de reconnaissance par leurs pairs de leur "expertise", dans la recherche de la force du marché et de négociation» comme le soutient Marchesnay (2002, p. 47). Ces derniers ont des formations et des expériences de cadres ou d'ingénieurs, ce qui leur permet d'adopter les attitudes propres aux entrepreneurs-managers qui cherchent à maximiser la valeur de l'entreprise et qui considère cette dernière comme un outil de travail. Le dirigeant adoptant une logique managériale s'attache à développer les compétences à travers des ré-allocations de ressources, voire d'activités, donc, à la seule exigence d'adaptabilité. Marchesnay 
ajoute en ce sens que «la logique d'action managériale se veut rationnelle», se basant sur la performance. Ce type de dirigeant recrute des cadres qui sont préparés à assurer la gestion rationnelle des ressources pour maximiser son profit. La conception managériale suppose l'existence de compétences clés qui permettent à l'entreprise de s'adapter et de reconfigurer sans cesse ses activités, en fixant comme cibles les entreprises les plus compétitives du secteur.

Enfin, les valeurs qui fondent la micromentalité «des dirigeants-entrepreneurs» leur donnent une dimension individuelle forte. En effet, leur micromentalité engendre une volonté de création et de croissance économique. Ainsi, rentabiliser et développer encore plus leurs entreprises (en taille, en chiffre d'affaires, en parts de marché) semblent être des objectifs très importants. Pour résumer, par leur soif de croissance et d'indépendance, les dirigeants se rapprochent du modèle des «nouveaux entrepreneurs» (Julien et Marchesnay, 1988), par l'importance qu'ils accordent à la production (création, amélioration, innovation, etc.), au développement de leurs activités, par leur négligence de la dimension patrimoniale de l'entreprise, ils se rapprochent aussi du «génial technicien» (Bauer, 1993) et par leur recherche d'expansion de leurs activités, de rentabilité à long terme (des produits et de l'entreprise) et des bonnes affaires, ils peuvent être qualifiés d'opportunistes ou rapprochés de «l'entrepreneur capitaliste» (Gresle, 1981; Zarca, 1986). Ainsi, leurs logiques se rapportant plus à leurs propres expériences et convictions produisent des comportements déviants de la rationalité de «l'homo-economicus».

Nous concluons ainsi cette section sur la diversité des typologies des dirigeants et l'existence de trois types de logiques d'action reconnus dans la littérature. En tenant compte de toutes les recherches déjà citées, nous allons résumer dans un tableau les traits qui permettent de reconnaître un dirigeant qui a fait appel à l'une ou l'autre des trois logiques d'action.

Nous adopterons ces trois logiques d'action pour la suite de notre recherche afin de détecter sur le terrain la logique d'action qui caractérise chaque dirigeant d'entreprise familiale étudiée. Nous vérifierons dans ce qui suit l'adoption des dirigeants d'entreprises familiales de logiques d'action différentes en nous référant à leurs choix stratégiques pour faire face au phénomène de la mondialisation. Nous vérifierons également si l'utilisation des cartes cognitives est une méthode appropriée dans notre cas pour faire ressortir les différentes logiques d'action des dirigeants d'entreprises familiales étudiées. 


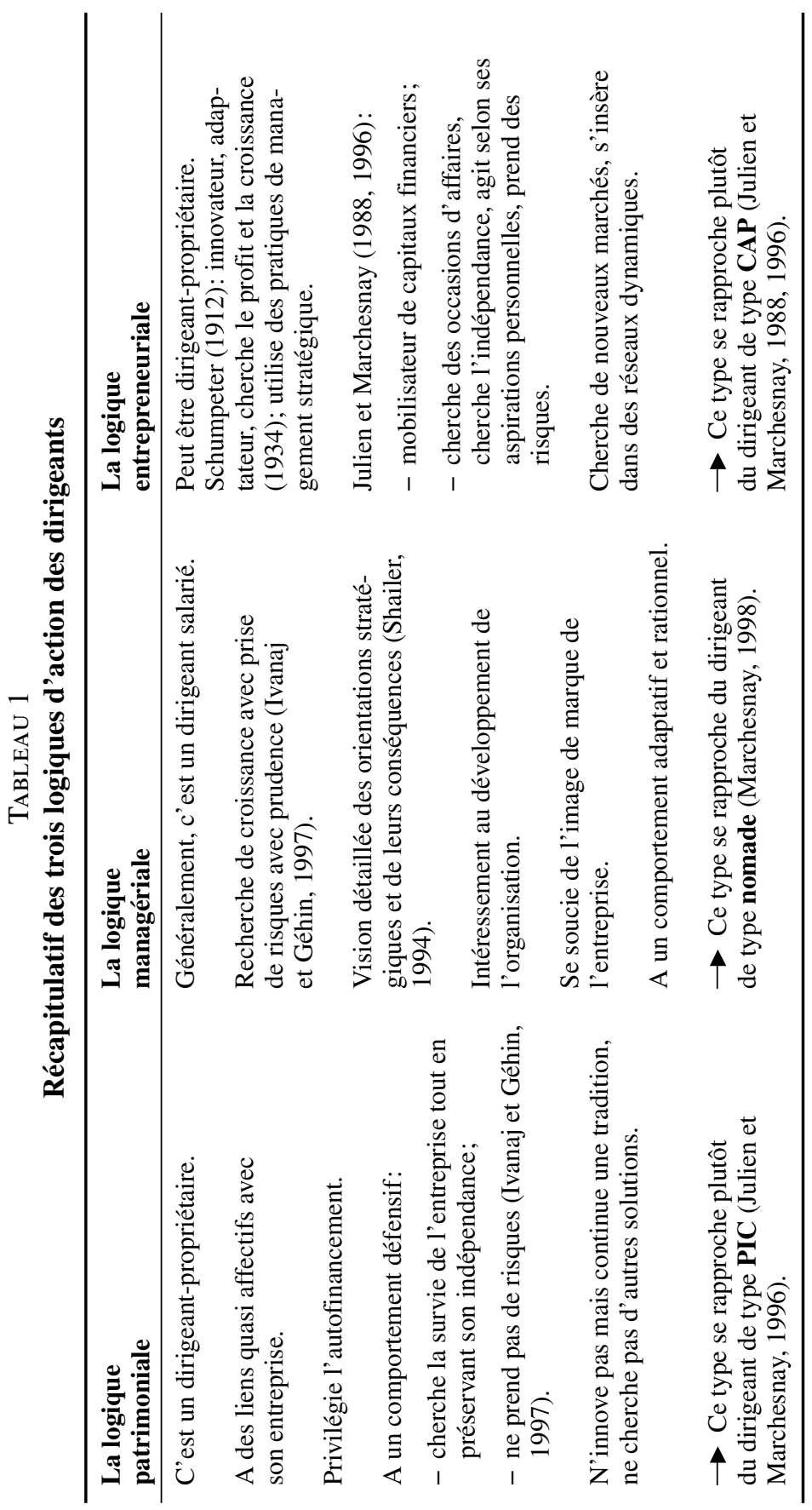

Revue internationale P.M.E., vol. 19, nº 2, 2006 


\section{La démarche méthodologique}

\subsection{Le contexte de la recherche}

Étant donné la difficulté d'accès à l'information dans les organismes publics et l'inexistence de statistiques concernant les entreprises familiales en Tunisie, nous avons préféré réaliser une pré-enquête, soit une prise de contact sur le terrain. La constitution du terrain de l'enquête a relevé d'un phénomène progressif de sélection, et ce, grâce à un questionnaire préliminaire auprès de 45 dirigeants d'entreprises appartenant au secteur de l'industrie chimique, à secteurs d'activité confondus, qui nous a permis de repérer 20 entreprises familiales ayant adopté des choix stratégiques différents pour réagir à la mondialisation. Cette pré-enquête a été réalisée auprès des entreprises implantées à Sfax, puisque cette ville est considérée comme étant le deuxième pôle industriel en Tunisie. Cette première phase de la recherche nous a permis de constituer une banque de données et de réduire la difficulté de sélection des entreprises familiales sur lesquelles portera notre recherche. Nous nous sommes fondée dans ce repérage des entreprises familiales sur la définition d'entreprise familiale que nous avons choisie préalablement. Nous cherchions simplement à repérer quelques entreprises familiales du secteur sur lesquelles nous pourrions mener notre recherche. Nous ne prétendons pas que les entreprises étudiées soient représentatives du secteur: elles constituent seulement des cas d'entreprises familiales qui sont étudiés pour la première fois en Tunisie en tant qu'entreprises familiales (voir tableau 2). Parmi les entreprises familiales repérées, 15 ont été finalement enquêtées, et ce, en nous fondant sur le principe de saturation. Étant donné l'objet de la recherche et son caractère exploratoire, nous avons choisi, pour réaliser notre partie empirique, seulement sept dirigeants d'entreprises familiales tunisiennes issues du secteur de l'industrie chimique. Nous avons décidé de limiter le nombre d'entreprises étudiées pour cette recherche pour des raisons de concision. L'étude des 15 cas est très longue et nécessite un travail que nous ne pourrons pas exposer en entier dans cet article et le secteur choisi constitue l'un des principaux axes de l'économie tunisienne. Ainsi, la valeur ajoutée de ce secteur représente $2 \%$ à $3 \%$ du PIB et $13 \%$ à $15 \%$ de la valeur ajoutée de la totalité des industries chimiques manufacturières ${ }^{3}$. Dans les industries chimiques, nous avons opté pour des industriels de parfumerie et cosmétiques, des fabricants de peintures et de colles, des fabricants transformateurs de plastique situés à Sfax.

3. Données fournies par la Fédération nationale de la chimie en Tunisie (2003) et tirées de l'étude stratégique sur les répercussions de la mise en place de la zone de libre-échange sur le secteur de la chimie en Tunisie, février 2000. 
TABLEAU 2

Données concernant les domaines d'activité choisis en Tunisie

\begin{tabular}{lccc}
\hline Domaines & $\begin{array}{c}\text { Parfumeries } \\
\text { et cosmétiques }\end{array}$ & Peintures/colles & $\begin{array}{c}\text { Fabricants } \\
\text { transformateurs } \\
\text { de plastique }\end{array}$ \\
\hline Statistiques & 30 & 40 & 420 \\
Nombre d'entreprises tunisiennes & LN & SOT, SIF, HEC & COP, EP, PS \\
\hline Les entreprises étudiées (Sfax) & & &
\end{tabular}

Source: Fédération nationale de la chimie (2001, 2003).

\subsection{Aperçu sur les entreprises familiales étudiées}

Pour des raisons de confidentialité, nous avons abrégé les noms des entreprises et de leurs dirigeants. Nous présenterons dans un tableau synoptique les critères qui déterminent la nature familiale de ces entreprises (voir le tableau 3).

Ce tableau montre, d'une part, que les entreprises étudiées sont de nature familiale et, d'autre part, que leurs dirigeants en sont tous les propriétaires. La majorité des informations qui figurent dans ce tableau sont tirées du questionnaire préliminaire passé dans le but de connaître les entreprises familiales du secteur. Signalons que ces informations se rapportent aux entreprises pendant la période des entrevues.

Les entreprises familiales repérées sont dirigées par des personnes qui sont partiellement ou totalement propriétaires. Leurs dirigeants ont tous été soumis aux mêmes supports d'entretiens et aux mêmes méthodes d'analyse de l'information collectée. Les entretiens ont été effectués avec prise de rendez-vous par appel téléphonique direct avec les dirigeants. Les interviewés ont été informés de la confidentialité des propos tenus.

\subsection{L'analyse des cartes cognitives}

Nous avons privilégié pour ce travail de recherche l'utilisation de la méthode qualitative et inductive. Nous adoptons la catégorie des enquêtes qualitatives basées sur les entretiens et interviews. Notre choix s'est fondé sur le fait que la méthode d'interview permet d'obtenir les informations recherchées d'une façon indirecte dans une relation de face à face avec l'interviewé. Afin d'obtenir de l'interviewé des expressions spontanées et des informations qui peuvent nous renseigner sur ses actions stratégiques, nous avons utilisé conjointement la méthode de l'interview non directive active et la technique des commentaires provoqués.

Mucchielli (1994, p. 28) note que dans l'interview non directive, «l'entretien est "ouvert" et "centré", c'est-à-dire qu'il repose non sur les réactions de l'interviewé à des questions précises mais sur l'expression libre de ses idées 


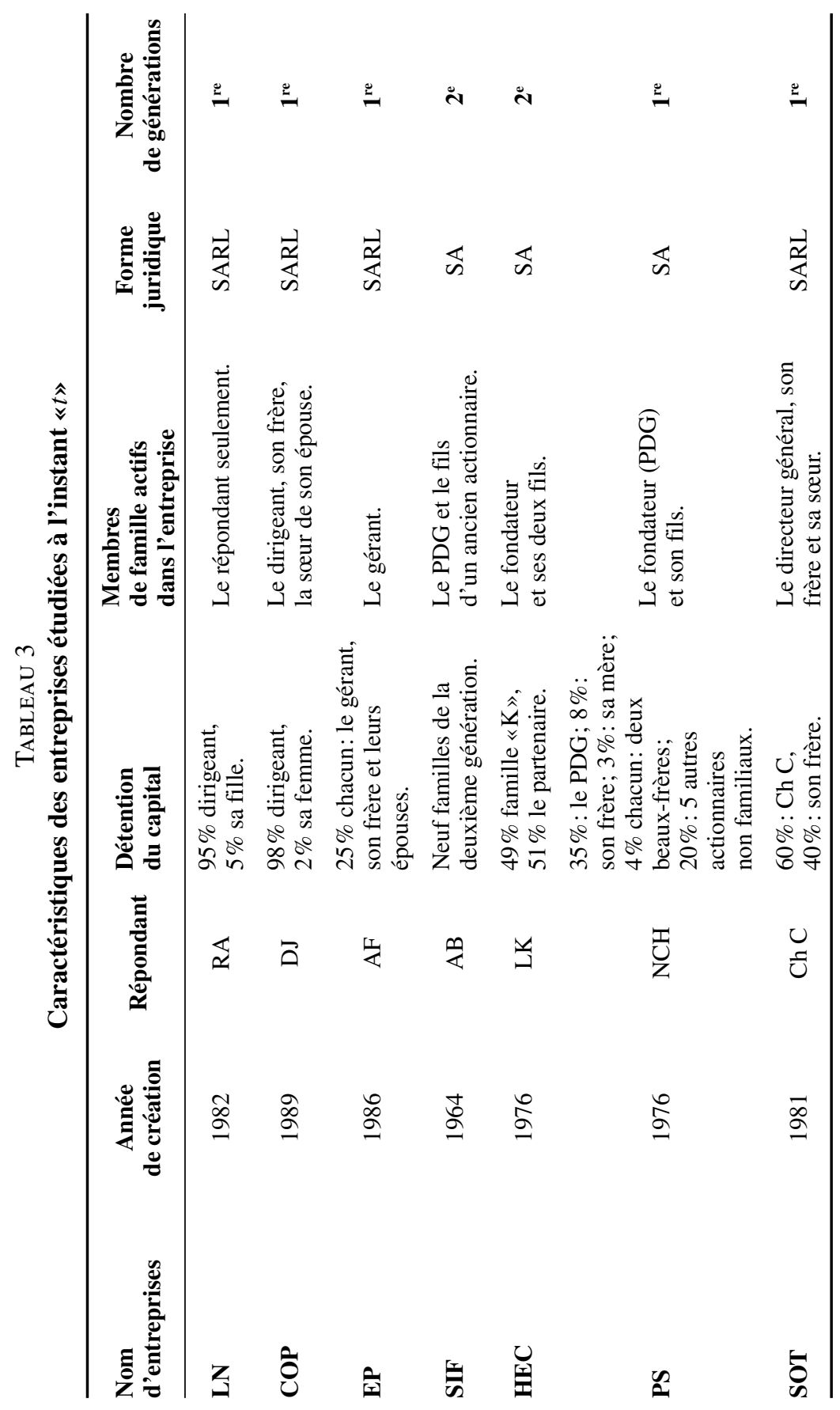

Revue internationale P.M.E., vol. 19, nº 2, 2006 
sur un sujet». Il ajoute, p. 30, que cette interview non directive est «active», car «l'interviewer soutient sans arrêt son interlocuteur dans sa réflexion, analyse et synthétise, au fil du discours, ce que dit l'interviewé d'important en essayant de ramener la compréhension de ce qui est dit par rapport à notre objectif». De plus, il précise, en ce qui concerne la technique des commentaires provoqués qu' «elle est utilisée dans l'interview individuelle» et cela correspond à notre cas puisque nous effectuons l'entretien avec le dirigeant de l'entreprise familiale. En l'occurrence, le chercheur présente à l'acteur ou aux acteurs sociaux, un petit cas, une situation problématique qui appelle de leur part des commentaires. Le matériel présenté aux enquêtés est appelé matériel «starter». Les sujets sont amenés à analyser le cas et à donner les prémisses de leur analyse, à exprimer leur opinion en justifiant celle-ci à l'aide de leurs valeurs. En employant cette méthode, nous avons mis à la disposition du dirigeant de l'entreprise familiale un texte comportant un cas fictif d'entreprise familiale que nous avons construit nous-même. Il correspond à un problème d'ordre que le dirigeant-propriétaire doit résoudre en prenant la décision qu'il juge la plus appropriée pour son entreprise. Nous avons invité les différents dirigeants individuellement à s'exprimer devant ce cas, en donnant leur avis sur le comportement du dirigeant de l'entreprise fictive et en soumettant leurs suggestions, tout en prenant la place du dirigeant du cas.

Nous avons pris contact avec les dirigeants par téléphone, pour prendre rendez-vous, en demandant de réaliser l'entrevue en fin d'après-midi de préférence pour ne pas que l'entretien soit interrompu par la secrétaire, un coup de téléphone ou une visite. Ces entrevues se sont déroulées dans les bureaux des dirigeants, un cadre dans lequel les dirigeants s'impliquent totalement, s'expriment librement et révèlent les informations importantes qui nous intéressent.

Durant les entretiens, nous avons adopté une attitude naïve, qui s'est traduite par des comportements non verbaux, une gestuelle dont le but est d'exprimer une activité intense d'écoute et une intention continue de comprendre la pensée de l'interviewé. C'est cette attitude qui a instauré le climat de confiance requis pour tenter de comprendre les dirigeants sans les juger. La durée des entrevues a varié d'une heure moins quart à trois heures, selon le dirigeant.

Puisque l'entreprise familiale «Houcine-Hema» (voir l'encadré à la page suivante) a un problème d'ouverture ou non à la mondialisation, nous avons demandé aux dirigeants ce qu'ils pensent de ce phénomène. Nous avons choisi de centrer le discours de chaque dirigeant autour de la mondialisation, c'est pour cela que nous avons placé ce concept «mondialisation» en haut de la carte cognitive. Toutes les «perceptions», «représentations», «convictions», «intentions» et «actions» ont été consignées aux endroits appropriés. Nous avons tenu compte des explications et du raisonnement suivi par chaque dirigeant pour réaliser la construction de sa carte cognitive. En effet, au fil du discours des dirigeants, nous avons synthétisé et essayé 


\section{Le matériel «starter» \\ Le problème de monsieur Hocine Hema}

Le groupe Hema ne cessait de prospérer et, paradoxalement, cela inquiétait fortement son p.-d.g., Hocine Hema. En effet, ses clients l'incitaient à développer son affaire à l'international: il venait de recevoir le matin même une offre extrêmement intéressante d'un grand distributeur de Hambourg, qui aurait souhaité monter une coentreprise avec son groupe, en apportant $51 \%$ du capital dans la filiale commune créée en Allemagne. Cette proposition lui semblait a priori honnête, mais elle posait la question du contrôle du capital de la société gérant un groupe de sociétés.

En effet, le groupe avait été créé par son père il y avait presque 50 ans maintenant et le capital avait été apporté par les membres du «clan Hema» dans la société gérant un groupe de sociétés. Le groupe s'était développé à partir de la société gérant un groupe de sociétés dans plusieurs directions, mais en se cantonnant dans le secteur de l'agroalimentaire. L'affaire avait suivi l'évolution des goûts du consommateur tunisien, à mesure que le niveau de vie s'était élevé et qu'une classe moyenne s'était constituée. Aujourd'hui, les consommateurs tunisiens étaient devenus très exigeants et ouverts aux produits étrangers. Par ailleurs, les produits fabriqués par les entreprises du groupe répondaient parfaitement aux exigences d'une clientèle musulmane, laquelle avait des difficultés à trouver certains de ces produits sur le marché européen, voire asiatique et américain.

Cependant, monsieur Hocine Hema savait que, sur le marché tunisien, il occupait pour l'instant une position dominante et plutôt protégée, finalement rentable, tout en sachant que la clientèle devenait très exigeante, risquant de devenir à terme moins fidèle aux marques du groupe, si des concurrents étrangers pénétraient sur le marché tunisien avec des marques connues internationalement.

Par ailleurs, il savait que, sur le marché international, des opportunités «fantastiques» s'offraient, comme il avait pu le constater lors de la foire de Hambourg, où il avait rencontré son collègue allemand. Mais il savait également que l'entrée sur les marchés mondiaux exigeait à la fois des capitaux, des connaissances et, plus généralement, une prise de risque élevée. Mais la survie du groupe Hema n'était-elle pas conditionnée à cet engagement dans l'international?

N.B.: Ce cas d'entreprise est fictif.

de ramener la compréhension de ce qui était dit par rapport à nos objectifs. Notre implication était totale lors des entretiens, puisque nous intervenions parfois pour manifester notre compréhension du contenu, pour recentrer le propos au moment opportun, pour reformuler les propos des dirigeants et reprendre le discours si nécessaire. Simultanément, nous procédions à l'analyse du contenu du discours, en donnant une première forme aux discours des dirigeants et en construisant les premières versions des cartes cognitives individuelles de ces derniers. Mucchielli (1994, 
p. 30-31) précise que «le chercheur est "actif" car il ne reporte pas à plus tard la compréhension de ce qui est dit sous prétexte qu'il enregistre tout. Il ajoute qu' «il ne s'agit donc pas d'enregistrer "bêtement" tout ce qui se dit pour l'analyser après coup, dans la tranquillité d'un bureau».

De retour à la maison, nous avons rempli une fiche qui reprend l'entretien, y compris le discours du dirigeant, pour nous assurer que tout ce qui avait été dit par le dirigeant figurait dans la carte et se trouvait au bon endroit.

À travers les petits détails dont nous nous sommes préoccupée pour mener à bien les entretiens avec les dirigeants des entreprises familiales, nous avons pu inciter les dirigeants à se projeter et à nous donner des informations mettant au jour leurs choix stratégiques: comment ils comptent s'y prendre et ce qu'ils ont entrepris comme actions stratégiques pour agir par rapport à l'ouverture des frontières. C'est ce qui a permis de révéler leurs logiques d'action.

Dans la majorité des cas, nous avons remarqué que les dirigeants commençaient à parler en prenant la place du dirigeant du cas, mais finissaient spontanément par nous parler de leurs propres expériences, celles de leur entreprise. C'est ainsi que nous avons pu avoir beaucoup plus de précisions sur leurs intentions et leurs actions stratégiques. Il ne faut pas oublier de signaler que, pendant les entrevues avec les dirigeants, et au fur et à mesure que les dirigeants se prononçaient, des relances neutres de conversations étaient engagées, soit pour comprendre ce qui avait été dit par les dirigeants, soit pour avoir plus de précisions concernant un point qui avait été évoqué rapidement par les dirigeants.

Le problème qui peut se poser dans cette deuxième phase de la recherche réside dans le décryptage du discours du dirigeant, qui souvent permet de révéler les faces cachées des intentions stratégiques. Marchesnay (2003b, p. 15) note que «le discours entend mettre en avant la clarté des intentions stratégiques du dirigeant, en quelque sorte, les yeux fixés sur l'horizon, sachant où il va et pourquoi». Dans le même ordre d'idées, Cossette et Audet (1994, p. 15) précise que «c'est le discours produit par un sujet dans un contexte d'interlocution particulier à partir de ses propres représentations cognitives qui guide le chercheur dans la fabrication d'une carte cognitive». Marchesnay (2003b, p. 15) montre dans ce sens que nous pouvons penser que l'attitude du décideur peut varier selon le type de problème: cette attitude est déjà susceptible de se faire selon le moment, la disposition d'esprit, la survenance d'événements ou d'informations plus ou moins inopinée. Il ajoute que «le fait de focaliser son attention sur "un cas", un problème, facilite le processus d'"élucidation", voire d'exploitation, des diverses formes de connaissances». C'est pour ces raisons que nous avons choisi de connaître les intentions et les actions stratégiques des dirigeants et d'en déduire, grâce à la carte cognitive, leurs logiques d'action manifestées par leurs choix stratégiques dans un contexte de mondialisation. 


\section{Les résultats}

Une fois la première et la deuxième phases de la recherche achevées - étant entendu que la première correspond à la situation d'entretien et la seconde à la schématisation du discours recueilli -, nous arrivons à la troisième phase, qui consiste à traiter et à analyser le contenu des cartes cognitives individuelles. En effet, à partir du matériel obtenu des entrevues «les cartes cognitives et les fiches d'entretien», nous avons pu interpréter les discours des dirigeants et analyser leurs cartes cognitives.

L'obtention des résultats de cette phase de la recherche nous permettra d'effectuer un retour sur le terrain pour vérifier nos résultats. Mucchielli (1994, p. 18) précise que «contrairement à ce qui se passe dans le modèle du fait scientifique dans les sciences exactes où l'objet reste l'objet, et même s'objective de plus en plus par la formulation même de l'esprit scientifique, les études qui se fondent sur une méthode qualitative présentent la possibilité de changement de l'observation qui transforme par conséquent les comportements et modifie les résultats». C'est pourquoi il nous a semblé important de retourner sur le terrain pour soumettre aux dirigeants les résultats les concernant et enregistrer leurs réactions. À travers ce retour sur le terrain, nous espérons une acceptation interne, c'est-à-dire, au sens de Mucchielli (1994, p. 18), «les résultats de la recherche reconnus comme pertinents par les acteurs sur lesquels porte la recherche», qui validera la méthode qualitative utilisée pour résoudre notre problématique de recherche. D'ailleurs, Zajc (1989) note que «le retour d'analyse est fondamental dans la mesure où une recherche qui ne serait pas considérée crédible sera discréditée par ses acteurs premiers: les participants à la recherche» (Mucchielli, 1994, p. 112).

\subsection{Les concepts émergents}

La littérature en cartographie cognitive suppose d'évaluer l'importance relative de chaque concept, et ce, à partir du nombre de facteurs auxquels un même concept est relié directement, soit comme facteur influençant, soit comme facteur influencé. Un concept est donc considéré comme particulièrement important lorsqu'il possède beaucoup de liens avec d'autres, ce que Nozicka, Bonham et Shapiro (1976) désignent par l'expression «cognitive centrality».

Nous réalisons une analyse qualitative qui consiste à repérer les concepts les plus importants et les idées dominantes et à relever les relations les plus émergentes. Pour cela, nous avons pris chaque carte cognitive et nous avons calculé manuellement le nombre de flèches reliées à chaque concept afin de connaître les concepts ayant le plus d'importance pour chaque dirigeant. Pour repérer dans chaque carte cognitive d'un dirigeant d'entreprise familiale, le ou les concepts les plus importants, nous avons identifié le nombre de flèches reliées directement, soit 
comme facteurs influençants, soit comme facteurs influencés (variables causes et variables conséquences). C'est ainsi que ressortent les concepts récurrents dans le discours du sujet, soit ceux qui semblent occuper une place privilégiée dans son mode de pensée.

TABLEAU 4

Les concepts émergents de l'analyse des cartes cognitives individuelles

\begin{tabular}{|c|c|c|}
\hline $\begin{array}{l}\text { Nom } \\
\text { d'entreprises }\end{array}$ & Les idées dominantes & Importance \\
\hline $\mathbf{L N}$ & $\begin{array}{l}\text { - L'entreprise est bien structurée. } \\
\text { - La qualité. } \\
\text { - La mise à niveau. } \\
\text { - Favorise les partenariats. }\end{array}$ & $\begin{array}{l}5 \\
5 \\
4 \\
4\end{array}$ \\
\hline COP & $\begin{array}{l}\text { - Augmentation de la concurrence. } \\
\text { - La mondialisation est une menace. } \\
\text { - La mondialisation bloque le développement. } \\
\text { - Avoir un bon service. }\end{array}$ & $\begin{array}{l}6 \\
5 \\
4 \\
4\end{array}$ \\
\hline EP & $\begin{array}{l}\text { - Favorise le partenariat. } \\
\text { - Encourage la mise à niveau. } \\
\text { - Considère la mondialisation comme une opportunité. }\end{array}$ & $\begin{array}{l}6 \\
5 \\
5\end{array}$ \\
\hline SIF & $\begin{array}{l}\text { - Il faut s’adapter. } \\
\text { - Il faut se préparer. } \\
\text { - Assure la compétitivité. }\end{array}$ & $\begin{array}{l}4 \\
4 \\
4\end{array}$ \\
\hline HEC & $\begin{array}{l}\text { - Permet le partenariat. } \\
\text { - La solidité financière. } \\
\text { - La notoriété de la marque. } \\
\text { - Bonne gestion. }\end{array}$ & $\begin{array}{l}8 \\
6 \\
6 \\
4\end{array}$ \\
\hline PS & $\begin{array}{l}\text { - Difficultés de pénétrer les marchés internationaux. } \\
\text { - La mondialisation n'encourage pas l'investissement. } \\
\text { - La mondialisation est une menace pour les entreprises } \\
\text { du tiers-monde. }\end{array}$ & $\begin{array}{l}5 \\
5 \\
5\end{array}$ \\
\hline SOT & $\begin{array}{l}\text { - La mondialisation est une bonne chose. } \\
\text { - La qualité. } \\
\text { - Avoir la certification ISO. }\end{array}$ & $\begin{array}{l}4 \\
4 \\
3\end{array}$ \\
\hline
\end{tabular}

Nous ne prenons en considération à ce niveau d'analyse que les concepts à hauts degrés d'importance. D'ailleurs, nous prenons à partir du plus haut degré d'importance les deux niveaux inférieurs, en nous fixant comme le minimum de degré d'importance (3), et ce, pour chaque dirigeant.

La présentation des concepts figurant dans les cartes cognitives montre que les dirigeants d'entreprises familiales tunisiennes réagissent différemment à la 
mondialisation et se dotent de logiques d'action différentes puisque ces derniers utilisent des concepts différents lorsqu'ils s'expriment sur le cas «Houcine-Hema». C'est ce que le tableau illustre en reprenant les concepts à hauts degrés d'importance pour chaque dirigeant.

Au regard du résultat obtenu concernant les concepts à hauts degrés d'importance, nous remarquons qu'à ce niveau d'analyse l'identification des concepts émergents et des idées dominantes nous renseigne sur la logique d'action de chaque dirigeant. Nous pensons que pour bien distinguer les choix stratégiques des dirigeants, nous devons nous intéresser à l'ensemble des concepts figurant dans les cartes cognitives, même ceux qui n'ont pas des degrés d'importance supérieurs. C'est l'ensemble des actions en cours de réalisation ou achevées et des intentions affichées qui divulguent mieux les différences dans les choix stratégiques des dirigeants.

\subsection{Identification des choix stratégiques}

Nous allons reprendre dans un tableau récapitulatif le discours de chaque dirigeant dans le but d'y mentionner tous les détails fournis par les interviewés en matière stratégique. Ce tableau récapitulatif va montrer le raisonnement adopté par chaque dirigeant grâce aux deux rubriques qui figurent dans le tableau et qui reprennent les intentions exprimées et les actions stratégiques menées par chaque dirigeant d'entreprise familiale. Toutes les informations qui y apparaissent sont reprises des cartes cognitives individuelles des dirigeants.

\section{TABLEAU 5}

Les discours des dirigeants des entreprises familiales illustrés dans les cartes cognitives

\begin{tabular}{ll}
\hline & \multicolumn{1}{c}{ L'entreprise familiale «LN» } \\
\hline Intentions & - Maximiser ses performances sur le marché avec de nouveaux supports \\
stratégiques & marketing. \\
& - Se préparer à l'export. \\
& - Profiter de l'effet d'image et de la réputation de la marque. \\
& - Étudier la proposition d'un partenaire étranger pour la fabrication \\
& d'une autre gamme de produits pour bébé. \\
& - Vendre pour l'Afrique la gamme de produits qu'il sous-traite. \\
\hline Actions & - A effectué le programme de mise à niveau. \\
stratégiques & - A obtenu l'ISO 9002. \\
& - A fait changer la dénomination de l'entreprise. \\
& - Effectue des études de marchés à l'étranger. \\
& - Dispose de plusieurs partenaires avec diverses formes de partenariat. \\
& - Crée un centre de formation bien équipé. \\
& - Organise des séminaires de formation animés par des techniciens étrangers.
\end{tabular}




\section{L'entreprise familiale «SOT»}

\begin{tabular}{ll}
\hline Intentions & - S'ouvrir sur l'étranger. \\
stratégiques & - Profiter de l'ouverture. \\
& - Se préparer à avoir la certification. \\
& - Se développer en changeant de domaines d'activité. \\
& - Disposer toujours des nouveautés de produits. \\
& - Préserver la confiance des fournisseurs, clients et tiers. \\
& - Vaincre la concurrence étrangère qui ne peut pas connaître le climat \\
& tunisien et les exigences de nos consommateurs mieux que nous. \\
\hline Actions & - Modernisation de l'entreprise. \\
stratégiques & - Participation à des foires spécialisées internationales. \\
\hline
\end{tabular}

\section{L'entreprise familiale «EP»}

\begin{tabular}{ll}
\hline Intentions & - Aligner les prix par rapport à la concurrence. \\
stratégiques & - Exporter plus ses produits, surtout vers les pays limitrophes, car ce \\
& sont des produits volumineux et aussi parce que l'acheminement se \\
& fait par voie terrestre qui est relativement bon marché. \\
& - Se préparer à toutes les éventualités en s'assurant de la qualité de \\
& son produit et en cherchant d'autres propositions d'autres entreprises \\
& étrangères pouvant procurer à son entreprise un plus. \\
\hline Actions & - A fait le programme de mise à niveau: matériel plus performant, à \\
stratégiques & grande cadence de production. \\
& - A conclu des accords avec des sociétés étrangères en louant leurs \\
& moules dans les périodes creuses du pays étranger. Cette location \\
& lui permet de fabriquer en sous-traitance des produits finis pour les \\
& vendre par la suite sur le marché local. \\
& L'entreprise bénéficie des moules, des matières premières, des équi- \\
& pements et du savoir-faire du partenaire étranger; en contrepartie, le \\
& partenaire gagne sur le volume de production et sur la diminution des \\
& coûts de transports et des frais de dédouanement. \\
- Fait de l'exportation à la Libye et à l'Algérie.
\end{tabular}

\section{L'entreprise familiale «PS»}

\begin{tabular}{ll}
\hline Intentions & - Assurer la compétitivité de son entreprise sur le local. \\
stratégiques & - Réduire au minimum les frais généraux. \\
& - Acheter de la matière première à faibles prix. \\
& - Recherche un partenaire solide qui accepte la minorité de la posses- \\
& sion du capital et qui propose un accord rentable et bénéfique. \\
\hline Actions & - Ont essayé de commencer le programme de mise à niveau qu'ils ont \\
stratégiques & aussitôt arrêté, car il coûte très cher. \\
& - Ont pensé à faire de l'exportation qui a été arrêtée en raison de diffi- \\
& cultés de paiement. \\
& - Font aujourd'hui de l'exportation indirecte: ils produisent des produits \\
& finis pour les vendre à leur tour à l'étranger. \\
& - Ont participé à plusieurs offres de partenariats qui n'ont pas abouti. \\
& - Ont contacté un fabricant d'automobiles comme partenaire, mais ils \\
& n'ont pas trouvé de terrain d'entente. \\
\hline & La société figure sur le site Internet du guide des foires internationales.
\end{tabular}




\section{L'entreprise familiale «HEC»}

\begin{tabular}{|c|c|}
\hline $\begin{array}{l}\text { Intentions } \\
\text { stratégiques }\end{array}$ & $\begin{array}{l}\text { - Devenir leader sur le marché local. } \\
\text { - Développer de nouveaux produits et de nouveaux marchés. } \\
\text { - Devenir centre de compétence pour la fabrication de la colle à base de } \\
\text { solvant pour l'Afrique, le Moyen-Orient et l'Europe du Sud. } \\
\text { - Exporter plus en développant de nouveaux marchés. } \\
\text { - Élargir ses gammes de produits en proposant constamment des } \\
\text { nouveautés. } \\
\text { - Impressionner la concurrence. } \\
\text { - Maintenir l'indépendance financière. }\end{array}$ \\
\hline $\begin{array}{l}\text { Actions } \\
\text { stratégiques }\end{array}$ & $\begin{array}{l}\text { - En 1993-1994, conclusion d'un partenariat avec des étrangers qui } \\
\text { assurent l'assistance technique. } \\
\text { - En 1997, partenariat avec un groupe multinational, leader mondial } \\
\text { dans ce secteur d'activité. } \\
\text { - A eu la certification ISO } 9002 \text {. } \\
\text { - Création de société de commercialisation. } \\
\text { - Innovation et diversification en continu. }\end{array}$ \\
\hline \multicolumn{2}{|r|}{ L'entreprise familiale «SIF» } \\
\hline $\begin{array}{l}\text { Intentions } \\
\text { stratégiques }\end{array}$ & $\begin{array}{l}\text { - Se développer en investissant en matière de recherche et développement. } \\
\text { - Réduire le coût de la non-qualité au minimum. } \\
\text { - Avoir un partenaire technique en versant en contrepartie des redevances. } \\
\text { - Créer d'autres entreprises dans d'autres créneaux. } \\
\text { - Se préparer aux éventualités en étant à l'écoute des consommateurs. }\end{array}$ \\
\hline $\begin{array}{l}\text { Actions } \\
\text { stratégiques }\end{array}$ & $\begin{array}{l}\text { - Création de sociétés de commercialisation. } \\
\text { - Création de société d'emballage pour produits alimentaires. } \\
\text { - Vendre à l'export pour les sociétés extraterritoriales. } \\
\text { - Certification ISO } 9002 \text { depuis } 1998 \text {. } \\
\text { - Préservation de la confiance des tiers dans le nom de l'entreprise, la } \\
\text { marque et le produit. }\end{array}$ \\
\hline
\end{tabular}

\section{L'entreprise familiale «COP»}

\begin{tabular}{ll}
\hline Intentions & - Être toujours à la page (technologie et gamme de produits). \\
stratégiques & - Suivre la demande des clients. \\
& - S'adapter aux exigences. \\
& - Se préparer pour faire face à la concurrence éventuelle. \\
& - Se développer malgré la situation économique internationale et \\
& survivre dans le contexte actuel. \\
& - Rester compétitif. \\
\hline Actions & - Assure de bonnes relations avec ses clients en se basant sur la \\
stratégiques & $\quad$ confiance en lui et en son produit. \\
& - Propose des prix compétitifs accompagnés de bonne qualité. \\
& - Assure un bon service de livraison, de délais de paiement et un service \\
& après-vente fiable et rapide. \\
& - Dispose d'une disponibilité de stock. \\
\hline
\end{tabular}


Ainsi, l'analyse effectuée des cartes cognitives illustrée dans ce tableau révèle que les cartes cognitives font apparaitre clairement les actions stratégiques mises en œuvre par les dirigeants et leurs intentions stratégiques. Dans notre cas, afin d'être fidèle au sens que le répondant accorde aux concepts qu'il utilise et pour limiter les effets de «contamination» au sens de Bougon (1992), nous avons repris les mots et les concepts utilisés par les dirigeants sans codification, ni synonyme. Nous obtenons ainsi des cartes cognitives individuelles «authentiques». En étant sûre de l'authenticité des informations existantes dans la carte cognitive et en essayant de reprendre exactement la logique suivie par le dirigeant pour tracer les liens entre les concepts, nous pouvons identifier facilement les stratégies adoptées par les dirigeants, notamment à l'échelle internationale et sur le local. Les cartes cognitives à notre disposition comportent divers concepts reflétant les stratégies adoptées par les dirigeants pour le marché international, en voici quelques-uns: se préparer à l'international, vendre plus à l'export, participation à des foires spécialisées internationales, certification ISO, création de sociétés de commercialisation, contrat de sous-traitance, contrat de partenariat, études de marchés à l'étranger, devenir centre de compétences au-delà des frontières, etc.

En élaborant le tableau 5, nous avons pu établir la logique d'action de chaque dirigeant. Pour ce faire, nous avons comparé pour chaque cas les critères de chaque dirigeant étudié en matière de choix stratégique et les caractéristiques définies dans la littérature, que nous avons exposées précédemment, concernant les logiques d'action reconnues par les gestionnaires.

En effet, chez le dirigeant de l'entreprise familiale $\mathbf{L N}$, on observe une attitude qui se rapproche plus de la logique d'action entrepreneuriale. Ce dirigeant est ouvert aux changements, prend des risques; il est prospecteur, innovant, toujours à la recherche de nouvelles occasions d'affaires pour faire des profits et assurer la croissance de son entreprise.

Le dirigeant de l'entreprise familiale COP appartenant à la première génération fait plutôt montre d'une logique d'action patrimoniale. En effet, il est contre les changements, il refuse les partenariats et le fait d'introduire un étranger à la famille dans l'entreprise. Il se fonde sur la confiance des clients en lui et en son produit pour préserver la part de marché de son entreprise sur le plan local. Il refuse la mondialisation bien qu'il affirme qu'elle ne dérange pas vraiment son secteur d'activité. Enfin, ce dirigeant a l'intention de s'adapter aux exigences locales pour se développer sur le marché tunisien sans prise de risque.

Le dirigeant de l'entreprise familiale EP prend des risques en investissant dans du matériel plus performant et à grande cadence. Il profite de la mondialisation (contrat de sous-traitance, des accords avec des sociétés italiennes); il cherche à 
maximiser le profit à travers la diversification dans les domaines d'activité; il est à la recherche de nouvelles occasions d'affaires pouvant procurer à son entreprise «un plus». Ce dirigeant manifeste une logique d'action plutôt entrepreneuriale.

Le dirigeant de l'entreprise familiale SIF s'adapte à la situation actuelle. Il a l'intention de se préparer aux éventualités en étant à l'écoute des consommateurs. Il ne prend pas des risques importants puisqu'il vend à l'export pour les sociétés extraterritoriales et cherche un partenaire technique en versant en contrepartie des redevances. Il se soucie de l'image de marque de l'entreprise puisqu'il s'efforce de préserver la confiance des tiers dans le nom de l'entreprise, la marque et le produit. Enfin, il veut développer son entreprise, en investissant en matière de recherche et développement. Les compétences de ce dirigeant démontrent une logique d'action plutôt managériale.

Le dirigeant de l'entreprise familiale HEC innove et prospecte, prend des risques à travers la conclusion de partenariat majoritaire avec un groupe multinational, cherche l'indépendance financière, obtient la certification ISO 9002 pour pouvoir conquérir de nouveaux marchés. C'est un dirigeant ouvert aux changements et qui profite de la mondialisation. Nous le qualifions plus d'entrepreneurial.

Chez le p.-d.g. de la société familiale PS, on relève une logique d'action plutôt patrimoniale. En effet, il ne veut pas prendre de risques, puisqu'il a refusé toutes les occasions d'affaires qui se sont présentées à l'entreprise. Il s'efforce de s'adapter et de suivre, mais la peur de perdre le patrimoine familial et le contrôle de l'entreprise fait échouer ces tentatives.

Selon le dirigeant de l'entreprise familiale SOT, il faut se préparer pour faire face à la mondialisation. Il considère que cela est possible s'il dispose de techniciens compétents et de fournisseurs à jour. Il cherche le développement de son entreprise par la certification ISO et par la participation aux foires internationales spécialisées. Enfin, il ne prend pas énormément de risques puisqu'il essaye de répondre à l'exigence des clients et ne considère pas le partenariat comme la première solution pour la croissance; il a plutôt l'intention de changer de domaine d'activité ou de diversifier le sien. L'attitude de ce dirigeant révèle donc une logique d'action plutôt managériale.

En fait, il ressort des tableaux 4 et 5 que les dirigeants des entreprises familiales étudiées manifestent dans leurs choix stratégiques trois logiques d'action. 
TABLEAU 6

Les logiques d'action des dirigeants

\begin{tabular}{lccccccc}
\hline $\begin{array}{l}\text { Nom d'entreprises } \\
\text { Logique d'action }\end{array}$ & LN & COP & EP & SIF & HEC & PS & SOT \\
\hline $\begin{array}{l}\text { Plutôt patrimoniale } \\
\text { Plutôt entrepreneuriale }\end{array}$ & $*$ & & $*$ & & $*$ & & \\
Plutôt managériale & & & & $*$ & & & $*$ \\
\hline
\end{tabular}

Dans ce tableau, nous pouvons voir que nous avons affaire à deux dirigeants qui ont des logiques d'action plutôt managériales, trois dirigeants dont les logiques d'action sont plutôt entrepreneuriales et deux dirigeants qui manifestent des logiques d'action plutôt patrimoniales. Il faut signaler que nous avons remarqué l'existence de logiques d'action nuancées, autrement dit, nous avons trouvé des dirigeants qui s'approchent plutôt de la logique d'action patrimoniale mais qui parfois agissent modérément. C'est pour cela que nous parlons de tendance et de rapprochement à une des logiques d'action.

Chaque dirigeant d'entreprise, en visionnant sa carte cognitive, prend conscience de la stratégie qu'il adopte, que ce soit sur le plan local ou international, puisque toutes ses actions réalisées jusque-là sont regroupées dans un même espace et reliées de manière à faire ressortir sa logique d'action. Nous espérons à travers cette relation émergente faire de la carte cognitive un outil d'aide à la prise de décision pour les dirigeants.

\section{Conclusion}

L'objectif principal de cette recherche était de montrer, d'une part, l'existence de différences dans les logiques d'action des dirigeants d'entreprises familiales et, d'autre part, de vérifier que la carte cognitive peut être un moyen pertinent pour établir ces logiques d'action en nous fondant sur les choix stratégiques des dirigeants.

Comme nous avons pu le constater, la cartographie cognitive peut être fort intéressante pour l'entreprise. Elle permet au dirigeant de rectifier ses attitudes et de revoir son comportement stratégique, en plus d'améliorer les intentions affichées en se concentrant davantage sur sa vision stratégique. Ce support visuel qu'est la carte cognitive facilite l'analyse par le dirigeant de la situation de son entreprise et le pousse à se questionner sur certaines éventualités. De nouvelles perspectives peuvent apparaître et d'autres priorités peuvent être mises en lumière grâce à la schématisation des représentations et des actions. 
Eden et al. (1979) soutiennent que «les managers ne sont pas pleinement conscients des processus mentaux mobilisés dans leur démarche décisionnelle». D'ailleurs, Axelrod (1976, p. 3) écrit aussi que «les preneurs de décision, quoi qu'il en soit, ne se rendent pas nécessairement compte des raccourcis spécifiques qu'ils utilisent lorsqu'ils réfléchissent aux choix de politiques. Et même s'ils sont parfois conscients de leurs propres styles cognitifs, ils ne le sont probablement pas quand il s'agit des limites que ces styles imposent et de la façon dont celles-ci peuvent être déplacées.»

Cet outil peut servir notamment pour les tiers de l'entreprise, tel le consultant. Ce conseiller peut se fonder sur la carte cognitive du responsable d'un poste clé dans l'entreprise pour analyser son état, connaître ses actions et ses orientations stratégiques et détecter les défaillances et leurs provenances. Il peut ainsi aider le dirigeant à surmonter les obstacles qui limitent la croissance et le développement de son entreprise. Il peut également prendre connaissance, à partir de la carte cognitive du dirigeant, des points forts de l'entreprise et renforcer ainsi sa stratégie, lui donner un essor par ses conseils et sa maîtrise du secteur et du marché.

Enfin, cette recherche a permis de cerner les logiques d'action que les dirigeants des entreprises familiales peuvent adopter d'autant plus que, dans notre cas, tous les dirigeants interviewés sont propriétaires de leur entreprise. Alors que nous avons généralement l'impression que les dirigeants-propriétaires d'entreprises familiales adoptent en majorité la logique d'action patrimoniale, les résultats de cette recherche montrent que les dirigeants-propriétaires des entreprises familiales peuvent aussi se doter de logiques d'action entrepreneuriale ou managériale. 


\section{Annexe}

FIGURE 1

\section{La carte cognitive du dirigeant de l'entreprise $L N$}

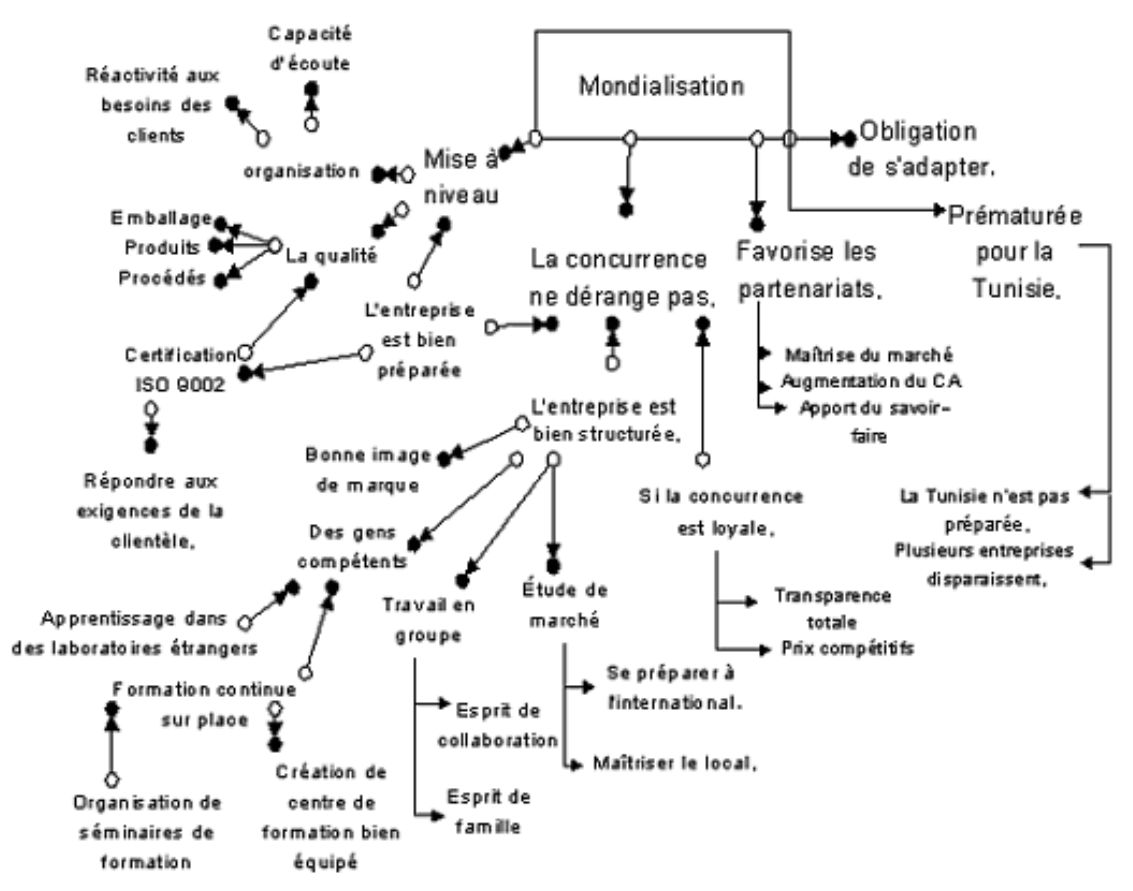

C Concept: flèche entrante

$\bullet$ Concept: flèche sortante

$\longleftrightarrow$ ou $\longleftarrow$ Concept : flèches entrantes

et/ou sortantes

Revue internationale P.M.E., vol. 19, nº 2, 2006

(C) 2006 - Presses de l'Université du Québec 
FIGURE 2

La carte cognitive du dirigeant de l'entreprise COP

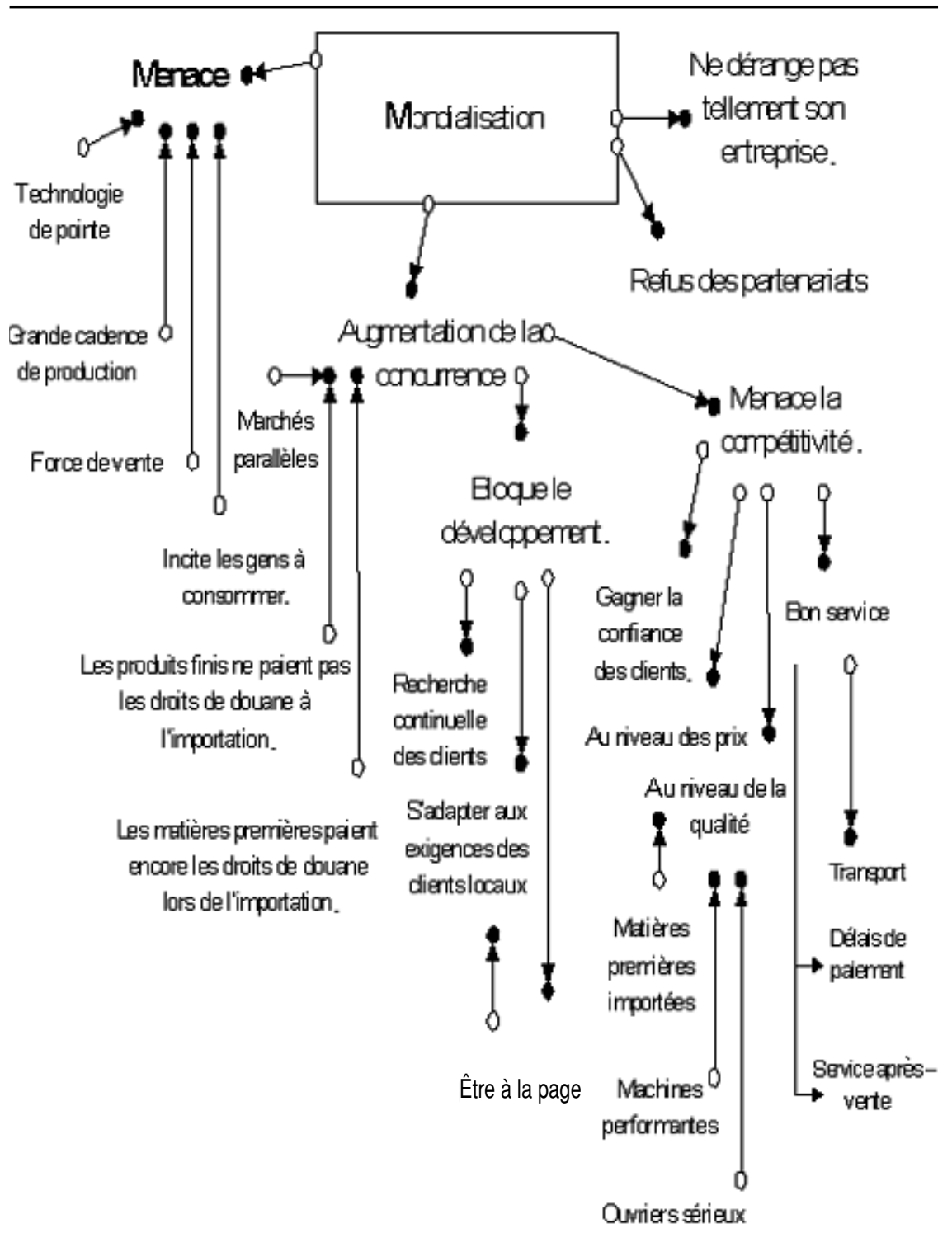

Revue internationale P.M.E., vol. 19, nº 2, 2006 
FIGURE 3

La carte cognitive du dirigeant de l'entreprise EP

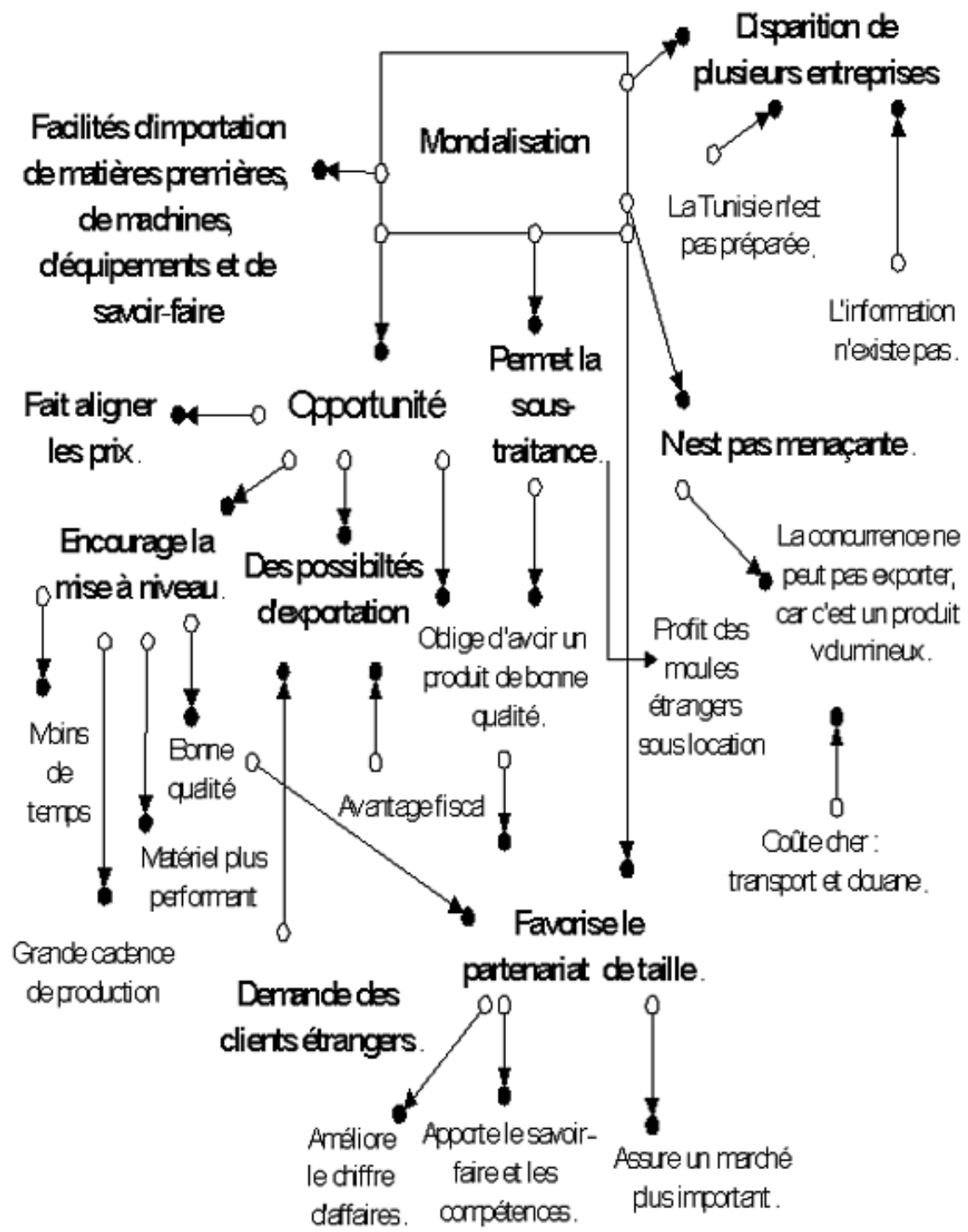


FIGURE 4

\section{La carte cognitive du dirigeant de l'entreprise SIF}

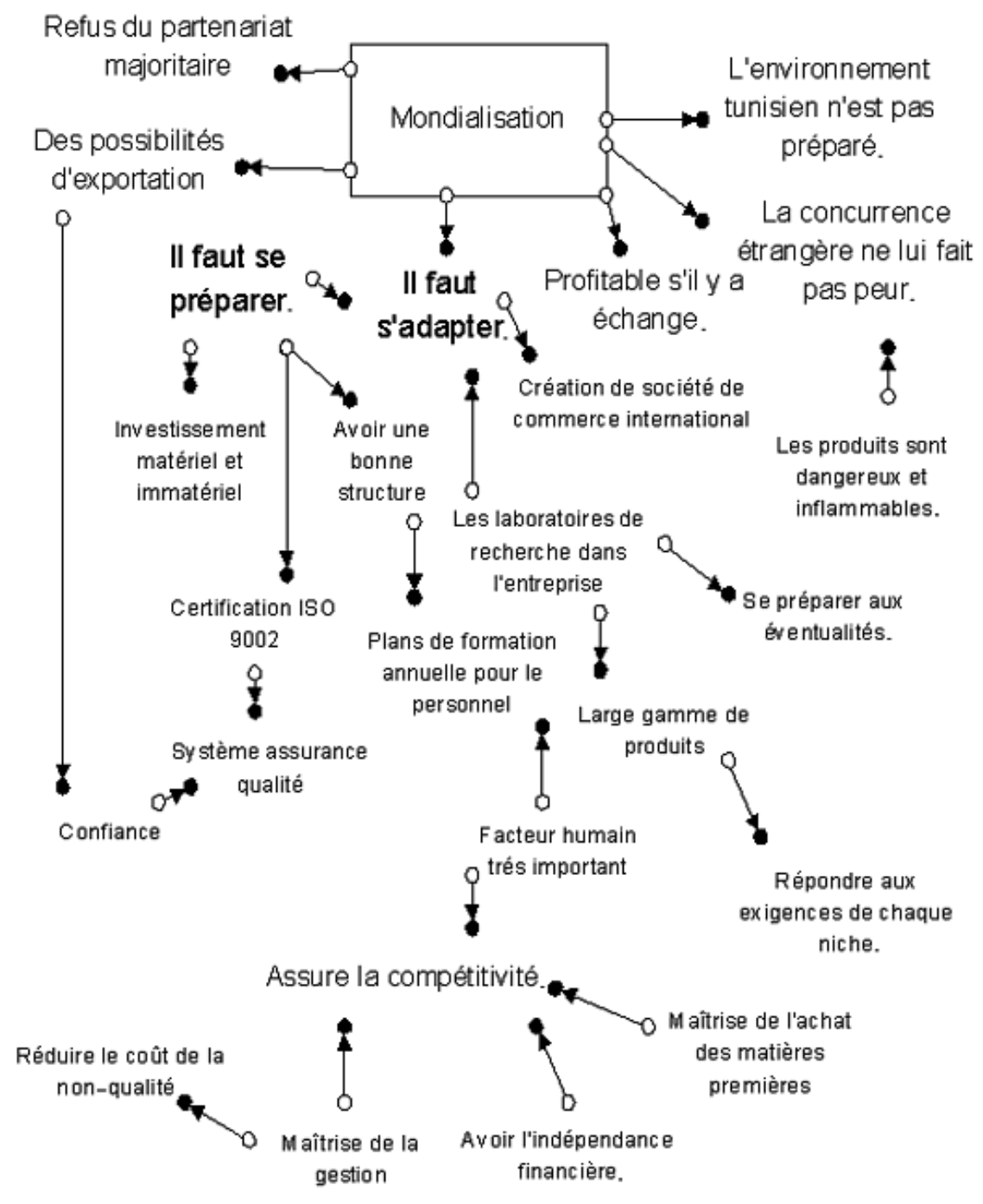

Revue internationale P.M.E., vol. 19, nº 2, 2006 
FIGURE 5

\section{La carte cognitive du dirigeant de l'entreprise HEC}

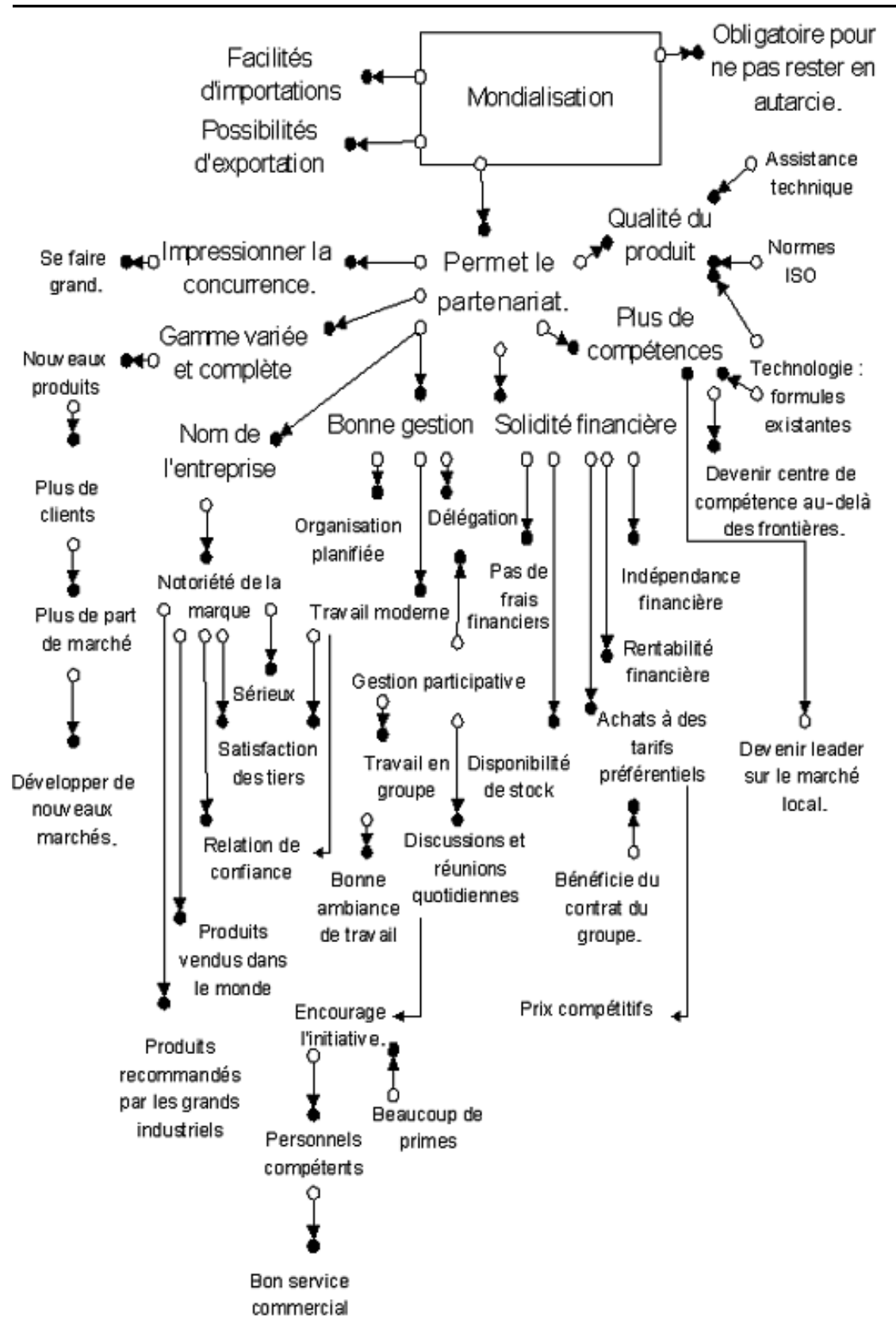

Revue internationale P.M.E., vol. 19, nº 2, 2006 
FIGURE 6

La carte cognitive du dirigeant de l'entreprise PS

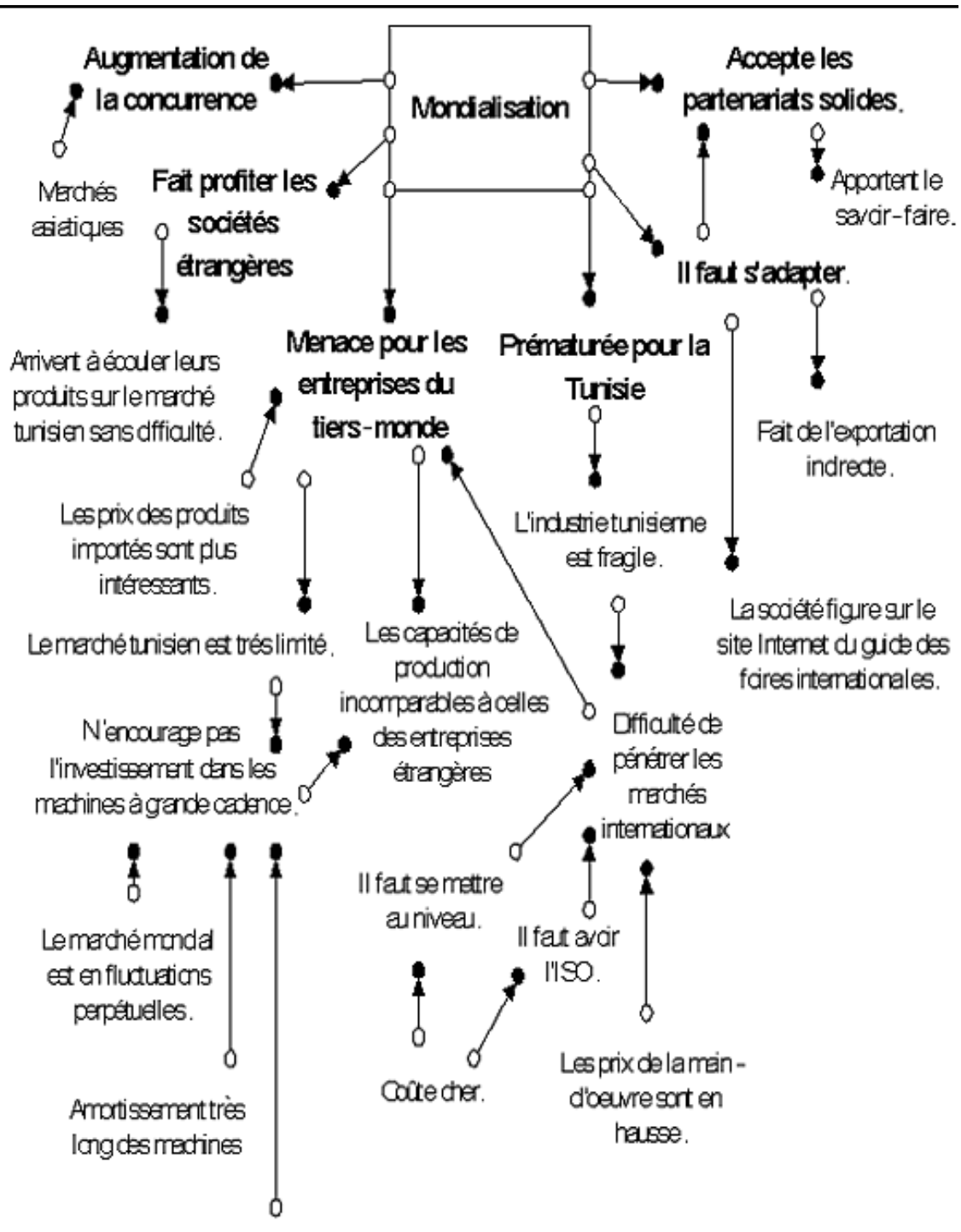

Lemerchén'e⿻ pes sûr. 
FIGURE 7

La carte cognitive du dirigeant de l'entreprise SOT

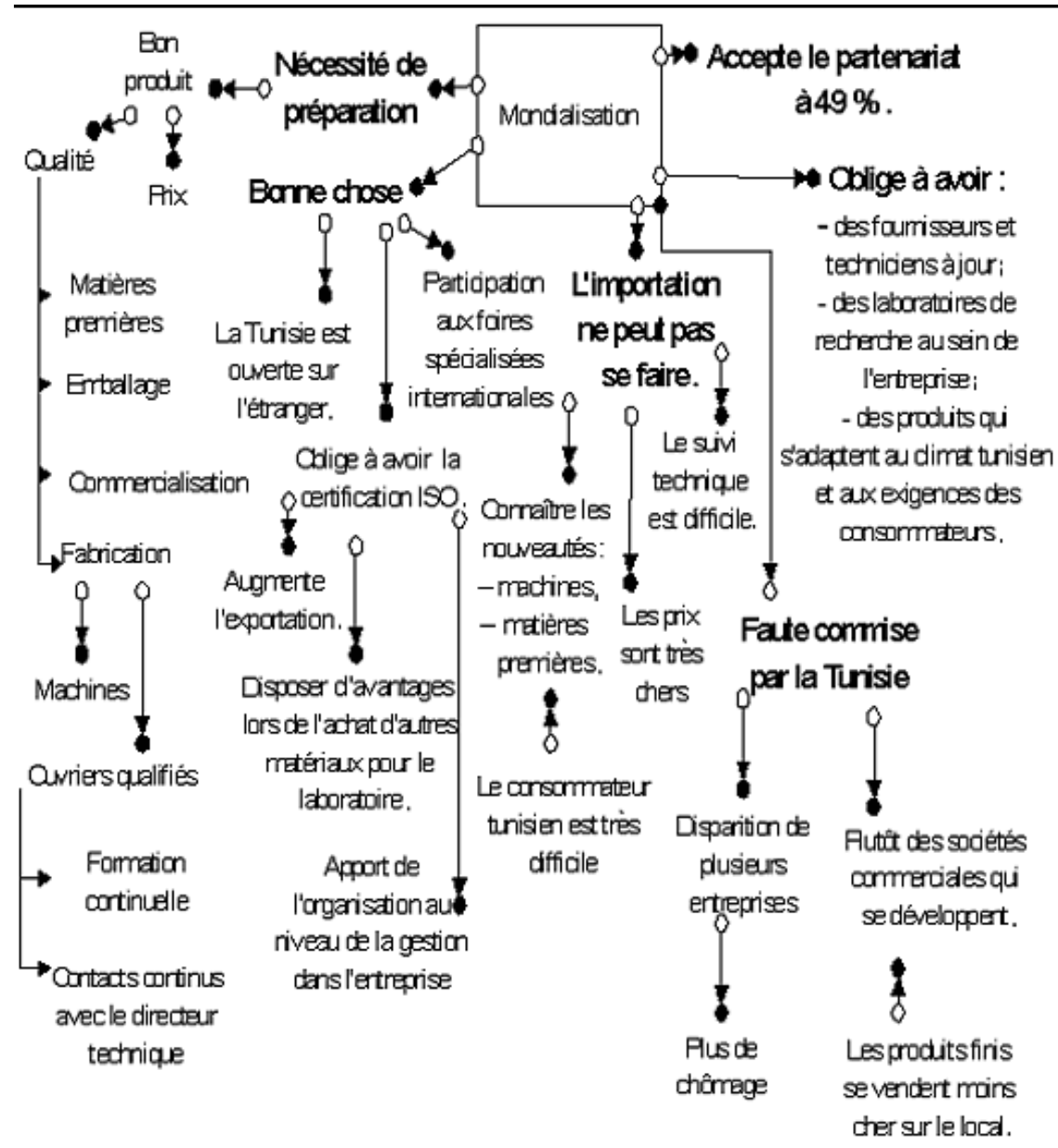




\section{Bibliographie}

Allain, S. (1999), «Approche cognitive de la gestion stratégique dans une entreprise monodécideur: le cas de l'entrepreneur agricole», Revue internationale PME, vol. 12, $\mathrm{n}^{\text {os }} 1-2$, p. 31-60.

AlLARD-PoÉsI, F. (1997), «De schèmes cognitifs individuels à un schème cognitif collectif: vers une perspective socio-cognitive», Actes de la IV Conférence internationale de Management stratégique, AIMS, vol. 1, 2-4 mai, Paris, p. 240.

Astrachan, J.H. et T.A. Kolenko (1994), «A neglected factor explaining family business success: human resources practices», Family Business Review, vol. 7, n 3 , automne, p. 251-262.

Axelrod, R. (1976), The Structure of Decision: The Cognitive Maps of Political Elites, Princeton, N.J., Princeton University Press.

Bauer, M. (1993), Les patrons de PME. Entre le pouvoir, l'entreprise et la famille, Paris, Inter Éditions.

Bougon, M.G. (1987), «The cause map analysis package. A still experimental suite of programs to analyse cause maps, especially equivocal ones», dans C. Rakotoarivelo (dir.), Carte cognitive et management: état de l'art, Actes de conférences, Université Stendhal-Grenoble, série: Recherche CERAG 96-01, décembre, p. 3-29.

Bougon, M.G. (1992), «Congregate cognitive maps: a unified dynamic theory of organization and strategy», Journal of Management Studies, vol. 3, n 29, p. 369-389.

Bougon, M.G., K.E. Weick et D. Binkhorst (1977), «Cognition in organizations: an analysis of Utrecht Jazz Orchestra», Administrative Science Quarterly, vol. 22, p. 606-639.

Bouhaouala, M. (1999), Micro-mentalités et logiques d'action des dirigeants des petites entreprises du tourisme sportif: contribution à une sociologie économique du sport, Thèse de doctorat, Université Joseph-Fourier, Grenoble I.

Bouhaouala, M. et P. Chantelat (2002), «Les pratiques commerciales des petites entreprises du tourisme sportif de nature: entre rationalité marketing et logiques sociales des dirigeants», Revue internationale PME, vol. 15, nº 1, p. 11-38.

Bowman, C. et G. JoHnson (1991), «Surfacing managerial patterns of competitive strategy: interventions in strategy debates», Communication présentée à la conférence de l'Academy of Management, Miami, Floride.

Carland, J.W., F. Hoy, W.R. Boulton et J.A.C. Carland (1984), «Differentiating entrepreneurs from small owners: a conceptualising», Academy of Management Review, vol. $9, \mathrm{n}^{\circ}$ 2, p. 354-359.

Catry, B. et A. Buff (1996), «Le gouvernement de l'entreprise familiale», Paris, PubliUnion Éditions.

Chabin, Y. (2001), La cohérence entre représentations de la performance et contrôle: le cas des entreprises intégrées de grande distribution alimentaire, Thèse de doctorat, Sciences techniques du Languedoc, Université de Montpellier II. 
Cossette, P. (1994a), «Les cartes cognitives au service de l'étude des organisations», dans P. Cossette, Cartes cognitives et organisations, Paris, Eska; Québec, Les Presses de l'Université Laval, coll. «Sciences de l'administration».

Cossette, P. (1994b), «La carte cognitive idiosyncrasique. Étude exploratoire des schèmes personnels de propriétaires-dirigeants de PME», dans P. Cossette, Cartes cognitives et organisations, Québec, Presses de l’Université Laval; Paris, Eska, p. 113-154.

Cossette, P. (1994c), «Développement d'une méthode systématique d'aide à la mise au point de la vision stratégique chez le propriétaire-dirigeant de PME: une étude exploratoire», Actes de la XXXIX ${ }^{e}$ Conférence mondiale de l'ICSB, du 27 au 29 juin, Strasbourg, p. 73-82.

Cossette, P. (1996), «La vision stratégique du propriétaire-dirigeant de PME: étude de cartographie cognitive», Revue internationale PME, vol. 9, nº 1, p. 123- 142.

Cossette, P. et M. Audet (1994), «Qu'est-ce qu'une carte cognitive?», dans P. Cossette, Cartes cognitives et organisations, Québec, Les Presses de l'Université Laval; Paris, Eska, coll. «Sciences de l'administration», chap. 2, p. 13-33.

Couret, M.A. (1993), «Le concept d'entreprise familiale et sa place dans les économies nationales et européennes», dans L'entreprise familiale en Europe, Annales de l'Université des Sciences sociales de Toulouse, Tome XLI, Toulouse, Les Presses de l'Université des Sciences sociales de Toulouse, p. 09-20.

D’Amboise, G. et Y. Gasse (1989), «Vision stratégique et activités de planification pour un modèle différent de recherche en $\mathrm{PME}$ », Communication présentée au XXXIV Congrès du Conseil international de la petite entreprise, juin, Québec.

Dunkelberg, W.C. et W.A.C. Cooper (1982), «Entrepreneurial typologies: an empirical study», Frontiers of Entrepreneurship Research, Wellesley, Mass., Babson College, p. $1-15$.

Eden, C. (1988), «Cognitive mapping: a review», European Journal of Operational Research, vol. 36, p. 1-13.

Eden, C., F. ACKermann et S. Cropper (1992), «The analysis of cause maps», Journal of Management Studies, vol. 29, n 3, p. 309-324.

Eden, C., S. Jones, D. Sims et H. Gunton (1979), «Images into models: the subjective world of the policy maker», Futures, vol. 11, n 1, p. 56-62.

Eden, C., S. Jones, D. Sims et T. Smithin (1981), «The intersubjectivity of issues and issues of intersubjectivity», Journal of Management Studies, vol. 18, n 1, p. 37-47.

Fahey, L. et V.K. NARAYANAN (1989), «Linking changes in revealed causal maps and environmental change: an empirical study», Journal of Management Studies, vol. 26, nº 4, p. 361-378.

FÉdération nationale de la Chimie (2001), Annuaire de la chimie et du plastique, UTICA, Tunisie.

Fédération nationale de la chimie (2003), Livret d'études, Élaboré par la Fédération sur le secteur du plastique, Tunisie.

GRESLE, F. (1978), Indépendant et petits patrons, pérennité et transformations d'une classe sociale, Thèse de doctorat, Université de Lille III. 
Gresle, F. (1981), «L'indépendance professionnelle», Revue française de sociologie, vol. 4, $\mathrm{n}^{\circ} 22$, octobre-décembre, p. 483-501.

GrizE, J.B. (1989), «Logique naturelle et représentations sociales», dans D. Jodelet, Les représentations sociales, Paris, Presses universitaires de France, chapitre 6, p. 153-168.

HADDADJ, S. et A. D'ANDRIA (1998), «Transmission internes et transmissions externes dans les PME françaises: existe-t-il des différences de changements stratégiques d'orientations stratégiques», Revue internationale PME, vol. 11, n 4 , p. 45-62.

HART, J.A. (1976), «Comparative cognition: politics of international control of the oceans», dans R. Axelrod (dir.), The Structure of Decision: The Cognitive Maps of Political Elites, Princeton, N.J., Princeton University Press, p. 180-217.

HaRT, J.A. (1977), «Cognitive maps of three Latin American policy makers», Work Politics, vol. XXX, no 1 , p. 115-140.

IvANAJ, V. et S. GÉHIN (1997), «Les valeurs du dirigeant et la croissance de la PME», Revue internationale PME, vol. 10, $\mathrm{n}^{\text {os }} 3-4$, p. 81-108.

JENKINS, M. (1994), «Creating and comparing strategic causal maps: issues in mapping across multiple organizations », Communication présentée à l'International Workshop on Managerial and Organizational Cognition, 26 et 27 mai, Bruxelles.

Julien, P.-A. et M. MARChESNAY (1988), La petite entreprise: principes d'économie et de gestion, Paris, Vuibert Gestion.

Julien, P.-A. et M. Marchesnay (1996), L'entrepreneuriat, Paris, Economica, coll. «Poche Gestion».

Kelly, G.A. (1955), The Psychology of Personal Constructs: A Theory of Personality, New York, Norton.

Kelly, G.A. (1963), A Theory of Personality: The Psychology of Personal Constructs, New York, Norton.

LANGFIELD-SMith, K. (1992), «Exploring the need for a shared cognitive map», Journal of Management Studies, vol. 3, $\mathrm{n}^{\circ} 29$.

LAUfER, J. (1975), «Comment on devient entrepreneur?», Revue française de gestion, $\mathrm{n}^{\circ} 2$, novembre, p. 11-23.

LAUKKANEN, M. (1994), «Comparative cause mapping of organizational cognitions», Organization Science, vol. 5, $\mathrm{n}^{\circ} 3$, août, p. 322-343.

McClelland, D.C. (1965), «Achievement and entrepreneurship: a longitudinal study», Journal of Personality and Social Psychology, vol. 1, p. 389-392.

Marchesnay, M. (1997), «La moyenne entreprise existe-t-elle?», Revue française de gestion, novembre-décembre, p. 85-94.

Marchesnay, M. (1998), «Confiances et logiques entrepreneuriales», Économies et Sociétés, Série «SG», n 25, p. 99-117.

MARChESNAY, M. (2002), «Pour une approche entrepreneuriale de la dynamique ressourcescompétences, essai de praxéologie», Les cahiers de l'ERFI, n 22.

Marchesnay, M. (2003a), «Petite entreprise et entrepreneur», Encyclopédie de la Gestion, $\mathrm{n}^{\mathrm{o}} 119$, p. 2209-2219. 
Marchesnay, M. (2003b), «Le discours stratégique au crible des modèles», Document de recherche, ERFI, février, p. 1- 21.

Miles, R.E. et C.C. Snow (1978), Organizational Strategy, Structure and Process, New York, McGraw-Hill.

Mucchielli, A. (1994), Méthodes qualitatives, Paris, Presses universitaires de France, coll.: «Que sais-je?», 128 p.

Nkongolo-Bakenda, J.M., G. D'Amboise et B. Garnier (1994), «L'association entre la manifestation de la vision stratégique des propriétaires-dirigeants de PME et la performance de leur entreprise», Revue internationale PME, vol. 7, $\mathrm{n}^{\circ}$ 1, p. 35-61.

Nozicka, G., G.M. Bonham et M.J. Shapiro (1976), «Simulation techniques», dans R. Axelrod, Structure of Decision: The Cognitive Maps of Political Elites, Princeton, N.J, Princeton University Press, p. 349-359.

O'Keefe, J. et L. NAdel (1978), The Hippocampus as a Cognitive Map, Oxford, Clarendon Press.

Rakotoarivelo, C. (1995), «Carte cognitive et management: état de l'Art», Actes de conférences, Université Stendhal-Grenoble, série: Recherche CERAG 96-01, décembre, p. 3-29.

ScheIN, E. (1983), «The role of the founder in creating organizational culture», Organizational Dynamics, American Management Association, vol. 12, nº 1, été, p. 13- 28.

Schumpeter, J.A. (1912), La théorie de l'évolution économique, Paris, Librairie Dalloz, p. 330-331, traduction de Theory of Economic Development.

SHAILER, G. (1994), «Capitalists and entrepreneurs in owner-managed firms», International Small Business Journal, avril-juin, vol. 12, n 3, p. 33-41.

Stubbart, C.I. et A. Ramaprasad (1988), «Probing two chief executives schematic knowledge of the U.S. Steel Industry using cognitive maps», dans. R. Lamb et P. Schrivastava (dir.), Advances in Strategic Management, Greenwich, JAI Press, p. 139-164.

VERSTRAETE, T. (1999), Entrepreneuriat: connaître l'entrepreneur, comprendre ses actes, Préface de Bertrand Saporta, Paris, L’Harmattan, coll. «Économie et innovation».

WeIcK, K.E. et M.G. BougON (1986), «Organisations as cognitive maps: charting ways to success and failure», dans H.P. Sims, J. et D.A. Gioia, The Thinking Organization: Dynamics of Organizational Social Cognition, San Francisco, Jossey-Bass, p. 102- 135.

ZaJC, L. (1989), «Les critères de rigueur de la recherche qualitative», Actes de colloque de la SOREAT.

ZARCA, B. (1986), L'artisanat français: du métier traditionnel au groupe social, Paris, Economica.

Revue internationale P.M.E., vol. 19, nº 2, 2006

(C) 2006 - Presses de l'Université du Québec

Édifice Le Delta I, 2875, boul. Laurier, bureau 450, Québec, Québec G1V 2M2 • Tél.: (418) 657-4399 - www.puq.ca

Tiré de: Revue internationale P.M.E., vol. 19, $\mathrm{n}^{\circ}$ 2, sous la direction de Louis Raymond - PME1902N

Tous droits de reproduction, de traduction et d'adaptation réservés 